Optimization of the safety factor profile for high noninductive current fraction discharges in DIII-D

J. Ferron, C. Holcomb, T. Luce, P. Politzer, F. Turco, A. White, J. DeBoo, E. Doyle, A. Hyatt, M. Murakami, T. Petrie, C. Petty, T. Rhodes, L. Zeng

January 21, 2011

Nuclear Fusion 
This document was prepared as an account of work sponsored by an agency of the United States government. Neither the United States government nor Lawrence Livermore National Security, LLC, nor any of their employees makes any warranty, expressed or implied, or assumes any legal liability or responsibility for the accuracy, completeness, or usefulness of any information, apparatus, product, or process disclosed, or represents that its use would not infringe privately owned rights. Reference herein to any specific commercial product, process, or service by trade name, trademark, manufacturer, or otherwise does not necessarily constitute or imply its endorsement, recommendation, or favoring by the United States government or Lawrence Livermore National Security, LLC. The views and opinions of authors expressed herein do not necessarily state or reflect those of the United States government or Lawrence Livermore National Security, LLC, and shall not be used for advertising or product endorsement purposes. 


\section{Optimization of the safety factor profile for high noninductive current}

\section{fraction discharges in DIII-D}

J.R. Ferron ${ }^{1}$, C.T. Holcomb ${ }^{2}$, T.C. Luce ${ }^{1}$, P.A. Politzer ${ }^{1}$, F. Turco ${ }^{3}$, A.E. White ${ }^{4}$, J.C. DeBoo ${ }^{1}$, E.J. Doyle ${ }^{5}$, A.W. Hyatt ${ }^{1}$, R.J. La Haye ${ }^{1}$, M. Murakami ${ }^{6}$, T.W. Petrie ${ }^{1}$, C.C. Petty ${ }^{1}$, T.L. Rhodes ${ }^{5}$, and L. Zeng ${ }^{5}$

${ }^{1}$ General Atomics, P.O. Box 85608, San Diego, California 92186-5608, USA

${ }^{2}$ Lawrence Livermore National Laboratory, 700 East Ave, Livermore, California 94550, USA

${ }^{3}$ Oak Ridge Institute for Science Education, Oak Ridge, Tennessee 37830-8050, USA

${ }^{4}$ Massachusetts Institute of Technology, 77 Massachusetts Ave, Cambridge, Massachusetts 02139, USA

${ }^{5}$ University of California-Los Angeles, PO Box 957099, Los Angeles, California 90095-7099, USA

${ }^{6}$ Oak Ridge National Laboratory, PO Box 2008, Oak Ridge, Tennessee 37831, USA e-mail contact of main author:ferron@fusion.gat.com

Abstract. In order to assess the optimum $q$ profile for discharges in DIII-D with $100 \%$ of the current driven noninductively $\left(f_{\mathrm{NI}}=1\right)$, the self-consistent response of the plasma profiles to changes in the $q$ profile was studied in high $f_{\mathrm{NI}}$, high $\beta_{\mathrm{N}}$ discharges through a scan of $q_{\text {min }}$ and $q_{95}$ at two values of $\beta_{\mathrm{N}}$. As expected, both the bootstrap current fraction, $f_{\mathrm{BS}}$, and $f_{\mathrm{NI}}$ increased with $q_{95}$. The temperature and density profiles were found to broaden as either $q_{\text {min }}$ or $\beta_{\mathrm{N}}$ is increased. A consequence is that $f_{\mathrm{BS}}$ does not continue to increase at the highest values of $q_{\text {min }}$. A scaling function that depends on $q_{\mathrm{min}}, q_{95}$, and the peaking factor for the thermal pressure was found to represent well the $f_{\mathrm{BS}} / \beta_{\mathrm{N}}$ inferred from the experimental profiles. The changes in the shapes of the 
density and temperature profiles as $\beta_{\mathrm{N}}$ is increased modify the bootstrap current density $\left(J_{\mathrm{BS}}\right)$ profile from peaked close to the axis to relatively flat in the region between the axis and the H-mode pedestal. Therefore, significant externally-driven current density in the region inside the $\mathrm{H}$-mode pedestal is required in addition to $J_{\mathrm{BS}}$ in order to match the profiles of the noninductive current density $\left(J_{\mathrm{NI}}\right)$ to the desired total current density $(J)$. In this experiment, the additional current density was provided mostly by neutral beam current drive with the neutral-beam-driven current fraction $40 \%-90 \%$ of $f_{\mathrm{BS}}$. The profiles of $J_{\mathrm{NI}}$ and $J$ were most similar at $q_{\min } \approx 1.35-1.65$, $q_{95} \approx 6.8$, where $f_{\mathrm{BS}}$ is also maximum, establishing this $q$ profile as the optimal choice for $f_{\mathrm{NI}}=1$ operation in DIII-D with the existing set of external current drive sources. 


\section{Introduction}

In a tokamak discharge with $100 \%$ noninductively driven current $\left(f_{\mathrm{NI}}=1\right)$ and a large fraction of self-generated bootstrap current [1] $\left(f_{\mathrm{BS}}\right)$, the safety factor $(q)$ profile plays a key role as a result of the close coupling to both the transport coefficients and the sources of noninductive current density [2]. The bootstrap current density $\left(J_{\mathrm{BS}}\right)$ is proportional to the local values of $q$ and the temperature and density gradients. Conversely, the $q$ profile is strongly dependent on $J_{\mathrm{BS}}$ when $f_{\mathrm{BS}}$ is large, and in a steady-state tokamak, $f_{\mathrm{BS}}$ must be large in order to minimize the required external current drive power [3]. The temperature and density gradients depend on the transport coefficients, which also depend on $q$ as well as the magnetic shear [4,5]. The stability limits to increasing pressure depend on the $q$ profile and the gradients [6,7]. Finally, the amount of current that can be driven by external sources depends on the temperature and density profiles [3]. The complexity of these interactions and of the underlying processes limits the present ability to predict the self-consistent density, temperature, and $q$ profiles using models.

In order to assess the optimum $q$ profile for $f_{\mathrm{NI}}=1$ discharges in DIII-D, the self-consistent response of the plasma profiles to changes in the $q$ profile was studied in high $f_{\mathrm{NI}}$, high normalized toroidal $\beta\left(\beta_{\mathrm{N}}\right)$ discharges. In a systematic scan of the minimum value of $q$ $\left(1<q_{\min }<2\right)$ and $q$ at $95 \%$ of the normalized poloidal flux $\left(4.5<q_{95}<6.8\right)$, the temperature and density profiles were measured and the inferred profiles of the noninductively driven current densities (bootstrap, neutral beam driven $J_{\mathrm{NBCD}}$, electron cyclotron driven $J_{\mathrm{ECCD}}$ ) and the noninductive current fractions were computed from models. Measurements were made at both $\beta_{\mathrm{N}} \approx 2.8$ and at the $\beta_{\mathrm{N}}$ achieved with the maximum available neutral beam heating power, 
$\beta_{\mathrm{N}}=3.1$ to 3.8. The $q$ profiles were monotonically increasing or had only small negative central shear, $q(0)-q_{\min }<0.5$, and large, localized, density or temperature gradients typical of internal transport barriers were not present. Here, the toroidal beta is $\beta=2 \mu_{0}\langle P\rangle / B_{\mathrm{T}}^{2}$ where $P$ is the plasma pressure, \langle\rangle indicates volume average, and $\beta_{\mathrm{N}}=\beta a B_{\mathrm{T}} / I_{\mathrm{p}}$ (with $\beta$ in $\%$, plasma current $I_{\mathrm{p}}$ in MA, minor radius $a$ in m, and vacuum toroidal magnetic field $B_{\mathrm{T}}$ in T).

As expected, both $f_{\mathrm{BS}}$ and $f_{\mathrm{NI}}$ increased with $q_{95}$. However,the temperature and density profiles were found to broaden as either $q_{\min }$ or $\beta_{\mathrm{N}}$ is increased. Because of these profile changes and the role of $q_{\min }$ in determining the total bootstrap driven current, the relation $[3,8-$ 10]

$$
f_{\mathrm{BS}} \propto \varepsilon^{0.5} \beta_{\mathrm{p}} \propto q_{95} \beta_{\mathrm{N}}
$$

( $\varepsilon=a / R$ where $R$ is the major radius, $\beta_{\mathrm{p}}=2 \mu_{0}\langle P\rangle /\left(\mu_{0} I_{\mathrm{p}} / l\right)^{2}, l$ is the path length around the plasma poloidal boundary) is not the best description of the results in these experiments. An alternative scaling function is presented here that depends on $q_{\min }$ and a measure of the pressure profile peaking in addition to $q_{95}$ and $\beta_{\mathrm{N}}$. As $\beta_{\mathrm{N}}$ was increased, the changes in the shapes of the density and temperature profiles were found to modify the $J_{\mathrm{BS}}$ profile from peaked close to the axis to relatively flat in the region between the axis and the H-mode pedestal. A peaked noninductive current density $\left(J_{\mathrm{NI}}\right)$ profile is required, however, to match the total current density $(J)$. Therefore, significant externally-driven current density in the region inside the H-mode pedestal is required in addition to $J_{\mathrm{BS}}$ to match the profiles of $J_{\mathrm{NI}}$ and the desired total $J$. These profiles were most similar at $q_{\min } \approx 1.35-1.65, q_{95} \approx 6.8$, where $f_{\mathrm{BS}}$ is also maximum, 
establishing this $q$ profile as the optimum choice for $f_{\mathrm{NI}}=1$ operation in DIII-D with the existing set of external current drive sources.

The achievable value of $\beta_{\mathrm{N}}$ is also a consideration in the choice of the optimum $q$ profile for steady-state operation. Operation at the highest $\beta_{\mathrm{N}}$ possible is preferred in order to maximize $f_{\mathrm{BS}}$ and fusion gain [3]. The stability limit to $\beta_{\mathrm{N}}$ tends to decrease as $q_{\text {min }}$ increases [6] (except in cases with particularly broad current profiles where the plasma can couple especially well to the conducting wall [11]). With fixed $\beta_{\mathrm{N}}$ and density and temperature gradients, $f_{\mathrm{BS}}$ would be expected to increase with $q_{\min }$. However, it is possible for lower values of $q_{\min }$ to allow access to the highest $f_{\mathrm{BS}}$ by way of improved stability. This was the case in the experiments described here, where the largest values of $\beta_{\mathrm{N}}$ and $f_{\mathrm{BS}}$ were obtained at the lowest values of $q_{\mathrm{min}}$ studied. In addition, broader density and temperature profiles (to achieve a broad pressure profile) increase the $\beta_{\mathrm{N}}$ stability limit $[6,7]$ but at the same time decrease the gradients and $J_{\mathrm{BS}}$. The broadest pressure profiles were found in this experiment at the highest values of $\beta_{\mathrm{N}}$ and the lowest value of $q_{95}$.

In previous experiments, many different $q$ profiles have been studied for use in advanced tokamak discharges with a high bootstrap current fraction [3,12-19]. These reports focused primarily on specific $q$ profiles where high-performance discharges were successfully produced. In contrast, here the $q$ profile was scanned over a broad range in order to explore in a systematic way how changes in the $q$ profile affect the plasma parameters fundamental to obtaining steadystate operation. In steady-state scenario research, the focus has tended to be on discharges with $q_{\mathrm{min}}$ as high as possible in order to reduce the poloidal field of the discharge center region, thereby increasing $J_{\mathrm{BS}}$ and $f_{\mathrm{BS}}$. Some approaches have monotonic or slightly reversed $q$ 
profiles, with $q(0)-q_{\min }<0.5$, while other approaches utilize negative central shear with larger values of $q(0)-q_{\min }$. In a negative central shear case there is typically a region near the midradius of the plasma with a relatively large pressure gradient and a local peak in $J_{\mathrm{BS}}$, commonly referred to as an internal transport barrier (ITB). An ITB can also be present in weak shear cases. In a discharge with $f_{\mathrm{NI}}=1$, it is essential that the total noninductive current density equal the current density required to maintain a stationary $q$ profile. Noninductive current drive that is too localized, such as $J_{\mathrm{NBCD}}$ near the axis resulting from high input power required to achieve the target $\beta_{\mathrm{N}}$ or $J_{\mathrm{BS}}$ in the region of an ITB, can be incompatible with steady-state operation. In addition, the large local pressure gradient in a discharge with an ITB can result in a $\beta_{\mathrm{N}}$ limit reduced below 3 [7]. The experiment reported here focused on weak shear configurations in order to study cases without an ITB with stable access to values of $\beta_{\mathrm{N}}$ near 4 .

The remainder of this report is organized as follows. The procedure followed for the experiment is described in section 2 . The measured density and temperature profiles as a function of the $q$ profile and $\beta_{\mathrm{N}}$ are described in section 3. The calculated bootstrap current is discussed in section 4 and the scaling of $f_{\mathrm{BS}}$ is discussed in section 5 . The total noninductively driven current is described in section 6 and there is a summary and discussion in section 7 . 


\section{Description of the Experiment}

The discharges for this experiment were created using the approach previously found to be optimum for high noninductive current fraction experiments in DIII-D [14]. The discharge shape was a double-null divertor slightly biased toward the top of the vacuum vessel in order to minimize the electron density by optimizing the use of the divertor cryopump capability. The discharge squareness was chosen to maximize the achievable $\beta_{N}$ [14]. Neutral beams at total power up to $13.5 \mathrm{MW}$ from five beam sources that inject in the direction of the plasma current were the primary external power source, with additional heating from gyrotron power (up to 3.2 MW) applied as electron cyclotron current drive deposited in the region $0.25<\hat{\rho}<0.6$ [14]. Here, $\hat{\rho}=\rho / \rho_{b}$ is the normalized plasma radius, where $\rho$ is the square root of the toroidal magnetic flux and $\rho_{\mathrm{b}}$ is its value at the discharge boundary.

The $q$ profile was varied by changing the values of $q_{95}$ and $q_{\min }$. The preprogrammed timing of the H-mode transition and the time evolution of $\beta_{\mathrm{N}}$ were varied during the discharge formation in order to change the value of $q_{\min }$ during the plasma current flattop [20-22]. The toroidal field was $2.0 \mathrm{~T}$, increased from $1.75 \mathrm{~T}$ used for previous $f_{\mathrm{NI}} \approx 1$ steady-state scenario experiments [14], in order to increase the achievable duration with $q_{\min }>2$. The total plasma current was varied at constant toroidal field in order to change the value of $q_{95}$, a reactorrelevant optimization method since fusion gain $Q$ increases strongly with $B$. Nine different $q$ profiles were studied, with $q_{\min } \approx 1,1.5$ and 2 and $q_{95} \approx 4.5,5.6$ and 6.8 (figure 1 ). Because the duration of the high $f_{\mathrm{NI}}$ phase of the discharge was at most $70 \%$ of the resistive diffusion time, the value of $q_{\text {min }}$ evolved slowly during the discharge from just above to just below the nominal value. 
In the figures in this report, scalar values such as $f_{\mathrm{BS}}, f_{\mathrm{NI}}$, and $f_{\mathrm{NBCD}}$ are displayed as a function of $q_{\text {core }}$, the average value of $q$ in the region $0.0<\hat{\rho}<0.3$, rather than as a function of $q_{\text {min }}$. Because the shape of the $q$ profile in the region $\hat{\rho}<0.3$ varies considerably, from monotonically increasing to flat or slightly decreasing, a single, local $q$ value such as $q_{\min }$ does not represent the scaling with $q$ of the total bootstrap current generated in the innermost portion of the discharge as well as an average $q$ value. $q_{\text {core }}$ is used in the scaling function for $f_{\mathrm{BS}}$ presented in section 5 .

Two separate sets of discharges were studied. In the first set, $\beta_{\mathrm{N}}$ was held approximately constant at 2.7-2.8 for up to $1.2 \mathrm{~s}$ (about $10 \tau_{\mathrm{E}}$, where $\tau_{\mathrm{E}}$ is the energy confinement time) through feedback control of the neutral beam power (with 8-10 MW required). The value of $\beta_{\mathrm{N}}$ was chosen to be low enough to allow discharges at all of the $q$ profiles to be produced without significant tearing mode activity. Nevertheless, the primary challenge in producing the discharges was avoidance of tearing modes, which was particularly difficult in the discharges with $q_{\min } \approx 2$. Only discharges without an $n=1$ tearing mode and with only low amplitude $n=2$ or $n=3$ modes were analyzed. Although some of the discharges studied had the reconstructed value of $q_{\min }$ near 1 , no evidence of sawtooth oscillations was found. All of the discharges at $\beta_{\mathrm{N}} \approx 2.8$ had the same injected ECCD power, $\approx 2.25 \mathrm{MW}$.

In the second set of discharges, the neutral beam input power was increased to the maximum available (13.5 MW). The duration of this high-power phase, $0.2-0.7 \mathrm{~s}$, was limited primarily by the total available neutral beam energy. Most of the discharges in this second set had similar values of $\beta_{\mathrm{N}} \approx 3.5$ (figure 2 ) with normalized confinement [23] $H_{98} \approx 1.5$. Two exceptions, the highest and lowest $q_{\min }$ discharges at $q_{95}=6.8$, achieved lower, 3.1, or higher, 3.8, values of 
$\beta_{\mathrm{N}}$ respectively. At $q_{\min } \approx 2, q_{95}=4.5$ no discharges were obtained at the maximum beam power without an $n=1$ tearing mode. No attempt to inject the maximum beam power was made at $q_{\min } \approx 2, q_{95}=5.6$. With the large $J_{\mathrm{NBCD}}$ near the axis generated by the high-injected power, the achieved $q_{\min }$ values in these highest $\beta_{\mathrm{N}}$ discharges tended to be somewhat below the nominal target values. The ECCD power varied between 1.5 and $3.2 \mathrm{MW}$ in this set of discharges.

The maximum $\beta_{\mathrm{N}}$ in the experiment was above the calculated ideal no-wall $n=1$ stability limit and close to the stability limit calculated with a perfectly conducting wall (figure 2). The ideal $n=1$ mode is expected to set the ultimate limit to $\beta_{\mathrm{N}}$ if resistive instabilities or higher- $n$ ideal modes do not limit $\beta_{\mathrm{N}}$ at a lower value. The highest $q_{\text {core }}$ discharges have somewhat reduced $\beta_{\mathrm{N}}$ stability limits, consistent with previous results on the scaling with $q_{\min }$ [6], but the ratio of the ideal-wall $\beta_{\mathrm{N}}$ limit to the experimental value, 1.05-1.25, shows no systematic scaling with the $q$ profile. In order to estimate the $\beta_{\mathrm{N}}$ at the stability limits, test equilibria were produced using the TEQ equilibrium code [24] with the same $q$ profile and discharge shape as in the experimental equilibria, but with the pressure profile scaled by a factor that is constant as a function of radius. For each new equilibrium, the $n=1$ stability was calculated using the DCON code [25] and the scale factor was iterated to find a marginally stable equilibrium.

The data presented in the figures in this report are the average of measurements obtained during the approximately constant $\beta_{\mathrm{N}}$ phase of each discharge and the error bars show the standard deviation during the averaging interval. At $20 \mathrm{~ms}$ intervals the plasma pressure was used together with magnetics and motional Stark effect (MSE) data in a complete equilibrium reconstruction [26] in order to obtain the flux surface geometry and $q$ profile. To supplement the 
MSE measurements during the equilibrium reconstruction, the $J$ profile in the H-mode pedestal region was constrained [6] to match the profile predicted by a time-dependent simulation of poloidal flux diffusion in the ONETWO [27] transport code using the measured temperature and density profiles. The plasma pressure was obtained using density profiles measured by Thomson scattering and $\mathrm{CO}_{2}$ interferometers; electron temperature from Thomson scattering and electron cyclotron emission; ion temperature, carbon impurity density and rotation velocity from charge exchange recombination; and the fast ion pressure profile calculated using the NUBEAM [28] package in the ONETWO code. The radial electric field contribution to the MSE measurements was accounted for using the radial electric field calculated from these profile data $[29,30]$. Finally, the reconstructed equilibria together with the measured temperature and density profiles were used in the ONETWO code without including poloidal flux diffusion to compute from models $J_{\mathrm{BS}}[31,32], J_{\mathrm{NBCD}}$ (using NUBEAM [28]) and $J_{\mathrm{ECCD}}$ (using TORAY-GA [33]).

The theoretical dependence of $J_{\mathrm{BS}}$ on the temperature, density, and $q$ profiles is well illustrated by the form of the model [31,32]. $J_{\mathrm{BS}}$ is directly proportional to the local value of $q$ and the total bootstrap current density is the sum of four terms, each proportional to the gradient of $n_{\mathrm{e}}, n_{\mathrm{i}}, T_{\mathrm{e}}$, or $T_{\mathrm{i}}$, the electron and ion densities and electron and ion temperatures, respectively.

$$
\left\langle\left\langle J_{\mathrm{BS}} B\right\rangle\right\rangle=-\frac{F q}{B_{\mathrm{T} 0} \rho}\left[T_{\mathrm{e}} \frac{\partial n_{\mathrm{e}}}{\partial \rho} \mathcal{L}_{31}+n_{\mathrm{e}} \frac{\partial T_{\mathrm{e}}}{\partial \rho}\left(\mathcal{L}_{31}+\mathcal{L}_{32}\right)+T_{\mathrm{i}} \frac{\partial n_{\mathrm{i}}}{\partial \rho} \mathcal{L}_{31}+n_{\mathrm{i}} \frac{\partial T_{\mathrm{i}}}{\partial \rho}\left(\mathcal{L}_{31}+\alpha \mathcal{L}_{34}\right)\right]
$$

Equation (2) is equation (5) from reference [31] rewritten using $q=B_{\mathrm{T} 0} \rho \partial \rho / \partial \psi$, where $\psi$ is the poloidal magnetic flux, $F(\rho)=R B_{\mathrm{T}}, B_{\mathrm{T} 0}$ is $B_{\mathrm{T}}$ at a reference major radius $R_{0}$, normally the center of the vacuum vessel, $\mathcal{L}_{31}, \mathcal{L}_{32}, \mathcal{L}_{34}$, and $\alpha$ are functions of the trapped particle fraction 
and the collisionality defined in reference [31], and $\langle\langle\rangle\rangle$ indicates flux surface average. Note that because the trapped particle fraction is zero on the axis in the approximation used here, $J_{\mathrm{BS}}$ always drops rapidly to zero between $\hat{\rho} \approx 0.1$ and the axis. 


\section{Density and Temperature Profiles}

The measured temperature and density profiles all have features with a systematic dependence on $q_{\mathrm{min}}, q_{95}$ or $\beta_{\mathrm{N}}$. These features are summarized in this section. The complete set of temperature and density profiles for the discharges in the $q$ profile and $\beta_{\mathrm{N}}$ scans is shown in figures 3 through 7 .

The electron and ion temperatures increase across the entire profile as $q_{95}$ decreases at constant $q_{\min }$ [figures 3(a), 4(a) and 5]. This is consistent with the increase in stored energy necessary to maintain a constant value of $\beta_{\mathrm{N}}$ as $I_{\mathrm{p}}$ is increased at constant $B_{\mathrm{T}}$ and with the increase in $\tau_{\mathrm{E}}$ with $I_{\mathrm{p}}$.

At constant $\beta_{\mathrm{N}} \approx 2.8$, the electron and ion temperature profiles broaden as $q_{\min }$ increases. This change is most clearly shown by the electron temperature profiles [figure 3(a)] where, at fixed $q_{95}$, the values at the axis and at the top of the H-mode pedestal are nearly independent of $q_{\text {min }}$ while the values at mid-radius increase significantly as $q_{\min }$ increases. The broadening is reflected in the electron temperature gradient [figure 3(b)]. In the region between the axis and the H-mode pedestal $(0<\hat{\rho}<0.8)$, the location of the peak in $\left|\partial T_{\mathrm{e}} / \partial \rho\right|$ shifts to larger radius as $q_{\min }$ increases and the peak value decreases. In addition to the profile broadening, in the two lowest $q_{95}$ cases the ion temperature profiles [figure $4(\mathrm{a})$ ] show a steepening of the profile at mid-radius at the highest $q_{\min }$. This is likely a result of a relatively broad region of reversed or small magnetic shear near the axis. Thus, in these two cases, the $q_{\min } \approx 2$ discharges have the highest values of $\left|\partial T_{i} / \partial \rho\right|$ [figure 4(b)] although the location of the peak still shifts to larger radius as $q_{\text {min }}$ increases. In the H-mode pedestal region $(0.8<\hat{\rho}<1.0),\left|\partial T_{\mathrm{e}} / \partial \rho\right|$ and $\left|\partial T_{\mathrm{i}} / \partial \rho\right|$ increase as 
$q_{95}$ decreases, reflecting an increased pedestal height, while there is no systematic trend with $q_{\min } \cdot$

With the maximum beam power, the temperature profiles are significantly broader than at $\beta_{\mathrm{N}}=2.8$ in the $q_{\min } \approx 1$ and $q_{\min } \approx 1.5$ cases (figure 5). As a result, the temperature profiles are nearly independent of $q_{\min }$. Except at mid-radius, the increase in $T_{\mathrm{e}}$ with the beam power is relatively small while $T_{\mathrm{i}}$ increases across the entire profile. The exception is the case at $q_{\min } \approx 1$, $q_{95}=4.5$ where the $T_{\mathrm{i}}$ increase is small and $T_{\mathrm{e}}$ decreases slightly on-axis and at the pedestal while increasing at mid-radius.

The pumping of the particle exhaust in the divertor region results in relatively low pedestal density, peaked density profiles, and little systematic dependence on the $q$ profile. At $\beta_{\mathrm{N}} \approx 2.8$ [figure 6(a)], in the region nearest the axis $(0<\hat{\rho}<0.5), n_{\mathrm{e}}$ is highest at the lowest values of $q_{95}$ at a given $q_{\min }$, but there is no consistent dependence on $q_{\min }$. The lowest values of $n_{\mathrm{e}}$ in the pedestal are at the highest value of $q_{\min }$, but there is otherwise no systematic dependence on the $q$ profile. The density gradient magnitude [figure $6(\mathrm{~b})]$ is peaked near $\rho \approx 0.2$, and is noticeably larger for $q_{\min } \approx 1$. Otherwise the shape of $\partial n_{\mathrm{e}} / \partial \rho$ is approximately independent of the $q$ profile.

When $\beta_{\mathrm{N}}$ is increased to the maximum possible with the available neutral beam power, the electron density increases, most noticeably at the H-mode pedestal, and the profiles broaden (figure 7). The largest change is at $q_{95}=4.5$ where the peak magnitude of the gradient in the region $0.0<\hat{\rho}<0.8$ decreased significantly [figure $7(\mathrm{~b})$ ]. As a result, at the higher $\beta_{\mathrm{N}}$ values, $\left|\partial n_{\mathrm{e}} / \partial \rho\right|$ increases as $q_{95}$ is increased. 
The ion density $\left(n_{\mathrm{i}}\right)$ is not directly measured but instead is inferred from charge balance using the measured $n_{\mathrm{e}}$ and carbon density profiles and the fast ion density profile computed using the NUBEAM model. The effective charge is in the range $1.9<Z_{\mathrm{eff}}<2.7$, with the highest $\beta_{\mathrm{N}}$ discharges having the highest values, with relatively uniform profiles in the region $0.0<\hat{\rho}<0.8$. The calculated fast ion density profile is peaked near the axis. The $n_{\mathrm{i}}$ profiles are, then, similar to, but less peaked than the profiles of $n_{\mathrm{e}}$. In fact, in the region $0<\hat{\rho}<0.8, \partial n_{\mathrm{i}} / \partial \rho$ is close to zero in the highest $\beta_{\mathrm{N}}$ discharges.

The key feature in the scaling of the density and temperature profiles is the broadening of profiles as a result of increases in either $q_{\min }$ or $\beta_{\mathrm{N}}$. This is reflected in the scaling of the peaking factor for the thermal pressure

$$
f_{\mathrm{p}}=\left[n_{\mathrm{e}}(0) T_{\mathrm{e}}(0)+n_{\mathrm{i}}(0) T_{\mathrm{i}}(0)\right] /\left\langle n_{\mathrm{e}} T_{\mathrm{e}}+n_{\mathrm{i}} T_{\mathrm{i}}\right\rangle,
$$

as shown in figure 8 , where $q_{\text {core }}$ has been used to characterize the value of $q$ in the region $0.0<\hat{\rho}<0.3$ (section 2). At $\beta_{\mathrm{N}} \approx 2.8$, the pressure is systematically less peaked at higher values of $q_{\text {core }}$ and the pressure peaking is significantly reduced at the maximum achieved $\beta_{\mathrm{N}}$. There is little dependence of the pressure peaking on $q_{\text {core }}$ in the high $\beta_{\mathrm{N}}$ cases as all of the profiles are relatively broad. The trends in the density and temperature profile shapes are also reflected in the $J_{\mathrm{BS}}$ profiles, as discussed in the next section. 


\section{Bootstrap Current Density}

Using the measured profiles of the density and temperature, profiles of the bootstrap current density were calculated as described in section 2 . In this section, these calculated profiles are compared as a function of the $q$ profile and $\beta_{\mathrm{N}}$. Calculated profiles are discussed because, although there is a method available that will derive a profile of $J_{\mathrm{NI}}$ from the experimental data [34], there is no way to obtain separate experimental profiles of the bootstrap, neutral beam driven and electron cyclotron driven components of the noninductive current density.

At $\beta_{\mathrm{N}} \approx 2.8$, the bootstrap current density is peaked near $\hat{\rho}=0.1$ [figure 9(a)]. The maximum $J_{\mathrm{BS}}$ is in the discharges at $q_{\min } \approx 1$ where the peak values of $\left|\partial n_{\mathrm{e}} / \partial \rho\right|$ are the largest (figure 6), but at mid-radius $(\hat{\rho}=0.5-0.7)$ these discharges have a small density gradient and thus the smallest $J_{\mathrm{BS}}$. In most of the region $0<\hat{\rho}<0.7$, at a given $q_{\min }, J_{\mathrm{BS}}$ is highest at the lowest value of $q_{95}$. This trend comes primarily from the two density gradient terms in equation (2) because of the increase in temperature as $q_{95}$ decreases. In the H-mode pedestal region there is no systematic variation of $J_{\mathrm{BS}}$ with $q_{\mathrm{min}}$ or $q_{95}$. In this region the larger temperature gradients at lower $q_{95}$ are compensated by the $q$ scaling of $J_{\mathrm{BS}}$.

At the $\beta_{\mathrm{N}}$ produced with the maximum available neutral beam power, the $J_{\mathrm{BS}}$ profile is significantly broader than at $\beta_{\mathrm{N}} \approx 2.8$ [figure $9(\mathrm{~b})$ ]. The peak values near $\hat{\rho}=0.1-0.2$ are reduced by up to a factor of 2.5 , and the bootstrap current density is increased at mid-radius. These changes are the result of broadening of both the temperature and density profiles. In the region of the $\mathrm{H}$-mode pedestal, unlike in the $\beta_{\mathrm{N}} \approx 2.8$ cases, there is a systematic increase of the width of the $J_{\mathrm{BS}}$ peak in the pedestal region with increasing $q_{95}$ that results primarily from an 
increase in the width of the electron and ion density gradient profiles. These profile width increases may be the result of increased $\beta_{p}[35]$.

The anticipated scaling of local values of $J_{\mathrm{BS}}$ with $q$ is not evident in the results for the experimental discharges (figure 9) because of the strong role of changes in the density and temperature profiles. This can be seen from a comparison of figure 9 with figure 10(a), which shows $J_{\mathrm{BS}}$ calculated with fixed density and temperature profiles so that it illustrates primarily the effect of a variation in the $q$ profile. In figure 10(a), the plasma can be roughly separated into two regions according to the scaling of $J_{\mathrm{BS}}$ with $q_{\mathrm{core}}$ or $q_{95}$. In the core region $(0.0<\hat{\rho}<0.3)$, figure 10(a) shows the expected scaling with $q_{\text {core }} . J_{\mathrm{BS}}$ is largest for the cases with $q_{\text {core }} \approx 2$, the opposite of what is shown in figure 9 (a). The curves in figure 10(a) in the region $\hat{\rho}>0.5$ are grouped according to $q_{95}$. In the H-mode pedestal region, $J_{\mathrm{BS}} \propto q_{95}$, unlike in figure 9(a) where $J_{\mathrm{BS}}$ is independent of $q_{95}$ and figure 9 (b) where the width of the current density peak increases with $q_{95}$. In figure $10(\mathrm{a}), J_{\mathrm{BS}}$ actually scales slightly more weakly than linearly with $q$ as a result of the weak scaling of the collisionality terms in equation (2) with $1 / q$. 


\section{Scaling of $f_{\mathrm{BS}}$}

The scaling relation given by equation (1) has been widely accepted for use in guiding the choice of parameter regimes for steady-state tokamak operation [3]. It points toward high values of $\beta_{\mathrm{N}}$ and $q_{95}$ in order to maximize $f_{\mathrm{BS}}$ and fusion gain. In this section, the results from the discharges in this $q$ profile scaling experiment are compared to this scaling relation and an alternative scaling function is proposed that provides a better match to the data.

The calculated bootstrap current fraction for the experimental data is maximum at the largest value of $q_{95}$ and the largest values of $\beta_{\mathrm{N}}$ (solid green circles, figure 11) in agreement with equation (1). A feature not included in equation (1), though, is that the variation of $f_{\mathrm{BS}}$ with $q_{\text {core }}$ is comparable to the variation with $q_{95}$. At $\beta_{\mathrm{N}} \approx 2.8$, the trend is for $f_{\mathrm{BS}}$ to increase with $q_{\text {core }}$ with the exception of two of the data points with $q_{\text {core }} \approx 2$, where the relatively high $q_{\text {core }}$ value is offset by broader temperature and density profiles and reduced gradients. At the maximum neutral beam power, the scaling of $f_{\mathrm{BS}}$ with $q_{\mathrm{core}}$ is affected by the scaling of $\beta_{\mathrm{N}}$ with $q_{\text {core }}$ [figure 2]. For $q_{95}=4.5$ and 5.6, $f_{\mathrm{BS}}$ increases from $q_{\text {core }} \approx 1$ to 1.5 . At $q_{95}=6.8$, though, $f_{\mathrm{BS}}$ decreases as $q_{\text {core }}$ increases as a result of the relatively low $\beta_{\mathrm{N}}$ achieved at the

highest $q_{\text {core }}$ and the relatively high $\beta_{\mathrm{N}}$ achieved at the lowest $q_{\text {core }}$. The ratio $f_{\mathrm{BS}} /\left(\varepsilon^{0.5} \beta_{\mathrm{p}}\right)$ (not shown) varies between 0.45 and 0.6 as a result of changes in the density and temperature profiles and $q_{\text {core }}$.

Values of $f_{\mathrm{BS}} / \beta_{\mathrm{N}}$ calculated from the experimental results (figure 12) differ in two key ways from the proportionality to $q_{95}$ predicted by equation (1). First, although the $f_{\mathrm{BS}} / \beta_{\mathrm{N}}$ values scale roughly linearly with $q_{95}$, the data extrapolate to a non-zero value at $q_{95}=0$. This 
reflects the importance of including $q_{\text {core }}$ in the scaling. Second, the data from the maximum beam power discharges lie systematically below the data from the $\beta_{\mathrm{N}} \approx 2.8$ discharges. This reflects the broadening of the density and temperature profiles when $\beta_{\mathrm{N}}$ increases. Note that the correct form of $\beta_{\mathrm{N}}$ for equation (1) is $\beta_{\mathrm{Nth}}$, the normalized toroidal $\beta$ calculated from the thermal plasma pressure [equation (3)], as used in figure 12. The component of the total plasma pressure from unthermalized neutral-beam-injected fast ions does not contribute to the bootstrap current density computed from equation (2). All current driven by the fast ion component is included in the NUBEAM model for $J_{\mathrm{NBCD}}$.

The following improved scaling relation eliminates both of the discrepancies with equation (1) by including both $q_{\text {core }}$ and $q_{95}$ and by including the thermal pressure peaking factor [equation (3)] to account for changes in the shapes of the temperature and density profiles.

$$
f_{\mathrm{BS}} / \beta_{\mathrm{Nth}}=A q_{\text {core }} f_{\mathrm{p}}^{\alpha_{\text {core }}}+B q_{95} f_{\mathrm{p}}^{\alpha_{95}} .
$$

In this function, the total bootstrap current is separated into a component in the innermost portion of the discharge that scales with $q_{\text {core }}$ and a component in the outermost portion that scales with $q_{95}$. There are separate exponents on the thermal pressure peaking factor for the two terms because, as $f_{\mathrm{p}}$ varies, the gradients in the innermost and outermost portions of the discharge change in opposite directions (thus the exponents should have opposite signs) and at different rates. As discussed in the appendix, a fitting function of this form can be obtained from equation (2).

The scaling function of equation(4) well represents the experimental data within the measurement uncertainties (figure 13). The uncertainties in the fitting coefficients (listed in the caption of figure 13) are large because the small amount of data and the size of the measurement 
uncertainties limits the capability to separate the scaling with the pressure profile shape from the scaling with $q_{\text {core }}$ and $q_{95}$. In addition, discharge-to-discharge differences in the details of the pressure profile shape are not included in equation (4). An F-test [36] showed that each of the fitting coefficients improves the fit by a statistically significant amount. Implicitly included in the fitting coefficients are the profiles of $\mathcal{L}_{31}, \mathcal{L}_{32}, \mathcal{L}_{34}$, and $\alpha$ [equation (2)] appropriate for the discharges in this experiment. Thus the form of equation (4) should be generally useful, but equation (2), which is easily evaluated, is preferable for the calculation of accurate values of $f_{\mathrm{BS}} / \beta_{\mathrm{N}}$ for an arbitrary tokamak.

The estimate of the fractions of the total bootstrap current $\left(f_{\mathrm{BS}} I_{\mathrm{p}}\right)$ in the innermost and outermost portions of the discharge from the two terms in equation (4) reflects the scaling of the temperature and density profile shapes with the $q$ profile and $\beta_{\mathrm{N}}$. At $\beta_{\mathrm{N}} \approx 2.8,40 \%-60 \%$ of the total bootstrap current is located in the innermost portion of the discharge. The largest fraction is at the lowest value of $q_{\text {core }}$ because in those cases the density profiles are especially peaked and because the temperature profiles broaden as $q_{\text {core }}$ increases. The smallest fraction is at the highest values of $q_{\text {core }}$ and $q_{95}$ where the temperature and density profiles are the broadest. In the higher $\beta_{\mathrm{N}}$ discharges, the fraction of the total bootstrap current in the innermost portion of the discharge drops to $20 \%-30 \%$ because of the broadening of the density and temperature profiles as $\beta_{\mathrm{N}}$ increases, and the variation with the $q$ profile is small because the density and temperature profiles have little dependence on the $q$ profile.

The largest fraction $(40 \%-55 \%)$ of the total bootstrap current results from the term in equation (2) that is proportional to $\partial T_{\mathrm{e}} / \partial \rho$. The $\partial T_{\mathrm{i}} / \partial \rho$ term accounts for $\approx 20 \%$, the $\partial n_{\mathrm{e}} / \partial \rho$ term $15 \%-20 \%$ and the $\partial n_{\mathrm{i}} / \partial \rho$ term $5 \%-20 \%$. This result differs from what would be expected 
from previous observations that the density gradient should be the most efficient generator of bootstrap current [31]. The $\partial T_{\mathrm{e}} / \partial \rho$ term is larger than the density gradient terms because in the outer half of the discharge the density gradient scale lengths are significantly larger than the electron temperature gradient scale length and, contrary to the assumption in reference [31], $\mathcal{L}_{32}>0$. The fraction of the total bootstrap current resulting from the temperature gradient terms increases with $\beta_{\mathrm{N}}$ as a result of the broadening of the density profiles. 


\section{Total Noninductively Driven Current}

It is the fraction of current driven by all noninductive methods and the degree of match of the shapes of the $J_{\mathrm{NI}}$ and $J$ profiles that determines whether a given $q$ profile can be maintained in steady-state. In these experiments, $J_{\mathrm{NI}}$ includes neutral beam driven current and electron cyclotron driven current in addition to the bootstrap current. Neutral beam driven current played a strong role as in some cases the fraction of neutral beam driven current $\left(f_{\mathrm{NBCD}}\right)$ was comparable to $f_{\mathrm{BS}}$. In this section, the profiles of $J_{\mathrm{NI}}, J_{\mathrm{BS}}, J_{\mathrm{NBCD}}, J_{\mathrm{ECCD}}$, and $J$ are compared and the features of the optimum $q$ profile for steady-state operation are discussed.

In almost all cases, the calculated $f_{\mathrm{NI}}$ increases with both $q_{\text {core }}$ and $q_{95}$ [figure $\left.14(\mathrm{a})\right]$ as a result of the combined changes in $f_{\mathrm{BS}}$ (figure 11) and $f_{\mathrm{NBCD}}$ [figure $14(\mathrm{~b})$ ]. Electron cyclotron current drive provided only $2 \%-4 \%$ of the total current because of the relatively high electron density. The NBCD model predicts little change in $J_{\mathrm{NBCD}}$ as the $q$ profile is varied with constant temperature and density profiles [figure 10(b)]. However, in the experiment $f_{\mathrm{NBCD}}$ increases with $q_{\text {core }}$, a result of higher $T_{\mathrm{e}}$ and reduced $n_{\mathrm{e}}$, and increases with $q_{95}$, the net result of $n_{\mathrm{e}}$ and $T_{\mathrm{e}}$ changes as $I_{\mathrm{p}}$ is varied at constant $B_{\mathrm{T}}$. There is a particularly large increase in $f_{\mathrm{NBCD}}$ as $q_{\mathrm{core}}$ is increased to the highest values $(1.7-2.1)$ that compensates in $f_{\mathrm{NI}}$ for the relatively low value of $f_{\mathrm{BS}}$ that is the result of relatively small density and temperature gradients (section 5). Thus the sole exception to the increase in $f_{\mathrm{NI}}$ with $q_{\text {core }}$ is the maximum neutral beam power discharge at $q_{\text {core }} \approx 1.77, q_{95}=6.8$ where $f_{\mathrm{NI}}$ is similar to the value in lower $q_{\text {core }}$ discharges as a result of relatively low $\beta_{\mathrm{N}}$ and therefore relatively low $f_{\mathrm{BS}}$. 
Comparison of the calculated $J_{\mathrm{NI}}$ to $J$ identifies the $q$ profiles that can best be maintained with noninductively driven current and thus are best suited to steady-state operation. The optimum $q$ profile is one with a high fraction of bootstrap driven current and a good match between the shape of the residual current density profile $\left(J-J_{\mathrm{BS}}\right)$ and the known methods to efficiently drive current by external means.

The highest bootstrap current fraction was found at the highest $\beta_{\mathrm{N}}$, the highest $q_{95}=6.8$ and $q_{\text {core }} \leq 1.5$. However, there is nothing that recommends one of the highest $\beta_{\mathrm{N}}$ discharges from the point of view of the shape of $J_{\mathrm{BS}}$ [figure 9(b)] in the region between the axis and the H-mode pedestal. In this region, the $J_{\mathrm{BS}}$ profiles in all of these discharges are relatively uniform. In the edge pedestal region there is a good match between $J_{\mathrm{BS}}$ and $J$ apart from a small ohmic current density that would not be present in a steady-state discharge.

A comparison of the profiles of $J$ and $J_{\mathrm{BS}}$ at $q_{95}=6.8$ (figure 15) shows a large difference in the region between the axis and the H-mode pedestal. Current driven by neutral beams injected at the discharge midplane provided most of the externally driven current needed to match $J-J_{\mathrm{BS}}$ because the $J_{\mathrm{NBCD}}$ profile is peaked near the axis and rather broad. The profile of $J_{\mathrm{BS}}+J_{\mathrm{NBCD}}$ has a shape that is a reasonably good match to the $J$ profile for the two cases with $q_{\text {core }} \geq 1.43$ [figure $15(\mathrm{~b}, \mathrm{c})$ ]. The residual current density is located in the region $0.2<\hat{\rho}<0.7$ where additional externally driven current of $\approx 20 \mathrm{~A} \mathrm{~cm}^{-2}$ would be required in order to reach $f_{\mathrm{NI}}=1$.

The match between $J_{\mathrm{NI}}$ and $J$ in the region $\hat{\rho}<0.2$ varies strongly with $q_{\text {core }}$. At $q_{\text {core }} \approx 1.1$ [figure $15(\mathrm{a})$ ] the residual current density is very large in this region both because $J$ 
is large and because relatively high $n_{\mathrm{e}}$ resulted in a relatively low peak value of $J_{\mathrm{NBCD}}$. Additional externally driven current in the region $\hat{\rho}<0.6$ with a peak value above $50 \mathrm{~A} \mathrm{~cm}^{2}$ at the axis would be required to match $J$ and $J_{\mathrm{NI}}$ at $q_{\text {core }}=1.1$. At $q_{\text {core }} \approx 1.77$ [Fig. 15(c)], $J_{\mathrm{NI}}$ exceeds $J$ near the axis because the neutral beam driven current is the largest at this $q$ profile and $J$ is reduced at higher $q_{\text {core }}$. Noninductive current overdrive near the axis $\left(J_{\mathrm{NI}}>J\right)$ will reduce $q_{\text {core }}$ in steady-state. This is consistent with the reduction in the maximum $q_{\text {core }}$ achieved in the experiment at $q_{95}=6.8$ from 2.1 to 1.77 as the neutral beam power was increased from the amount necessary to obtain $\beta_{\mathrm{N}}=2.8$ to the maximum available.

In order to operate the $q_{95}<6.8$ discharges at $f_{\mathrm{NI}}=1$, significantly more noninductively driven current than was obtained in this experiment is required and it must have a relatively broad profile. This is illustrated by the current density profiles for the $q_{\text {core }} \approx 1.56, q_{95}=4.5$ discharge (figure 16). The residual current density profile in this case is $\approx 40 \mathrm{~A} \mathrm{~cm}^{-2}$ at $\hat{\rho}=0.3$ and, although it decreases with radius, is still $\approx 20 \mathrm{~A} \mathrm{~cm}^{-2}$ at $\hat{\rho}=0.8$. Because of the relatively low $f_{\mathrm{NI}}$ and high $I_{\mathrm{p}}$, the total additional noninductively driven current that is required to obtain $f_{\mathrm{NI}}=1$ in this case is more than a factor of three above what is required for the cases shown in figure 15. The combination of the chosen value of $B_{\mathrm{T}}$ and the available neutral beam power was one factor that limited $f_{\mathrm{NI}}$ in this experiment. An overview of how to increase $f_{\mathrm{NI}}$ and $f_{\mathrm{BS}}$ at $q_{95}<6$ is included in section 7.

There is a significant gain in $f_{\mathrm{NI}}$ if the discharge can be operated at the same $\beta_{\mathrm{N}}$ but at reduced $n_{\mathrm{e}}$ and thus increased temperatures. If the density gradient is not changed, then $J_{\mathrm{BS}}$ would increase because the density gradient terms in equation(2) are multiplied by the 
temperature. The externally driven noninductive current density would increase. The potential gain at reduced $n_{\mathrm{e}}$ is illustrated by a test calculation for the $q_{95}=6.8, q_{\text {core }}=1.43$ discharge [figure 15(b)] which assumes a uniform $20 \%$ reduction in density at constant $\beta_{\mathrm{N}}$ (figure 17). In this test case, $f_{\mathrm{NBCD}}$ and $f_{\mathrm{ECCD}}$ both increased by $60 \%$ resulting in $f_{\mathrm{NI}}>1$. With the exception of the noninductive current overdrive in the region $\hat{\rho}<0.2, J_{\mathrm{NI}}$ is a good match to $J$. 


\section{Summary and Discussion}

Systematic changes in the shapes of the density and temperature profiles were found as $q_{\min }$ and $q_{95}$ were varied over the range of interest for tokamak operation with $100 \%$ of the current driven noninductively. There were also systematic changes as $\beta_{\mathrm{N}}$ was varied. The primary change was a broadening of the profiles as either $q_{\text {core }}$ or $\beta_{\mathrm{N}}$ was increased. In the $\beta_{\mathrm{N}} \approx 2.8$ discharges, as $q_{\text {core }}$ is increased, the electron and ion temperature profiles broaden as a result of an increase at mid-radius with little change near the axis or at the H-mode pedestal. With the increase in $\beta_{\mathrm{N}}$ to the value obtained with the maximum available neutral beam power, the temperature profiles become nearly independent of $q_{\text {core }}$ at a given value of $q_{95}$. The density profiles showed little systematic dependence on the $q$ profile except for relatively large gradient near the axis in the lower $\beta_{\mathrm{N}}$ discharges with $q_{\text {core }} \approx 1$. The increase in $\beta_{\mathrm{N}}$ resulted in broader density profiles primarily as a result of increased density at the H-mode pedestal.

The broadening of the density and temperature profiles as $q_{\text {core }}$ and $\beta_{\mathrm{N}}$ are increased reduces the gradients and, as a result, $J_{\mathrm{BS}}$. Thus the $J_{\mathrm{BS}}$ profiles are peaked near $\hat{\rho} \approx 0.1$ at $\beta_{\mathrm{N}} \approx 2.8$, but are much more uniform in the region inside the H-mode pedestal at the highest values of $\beta_{\mathrm{N}}$. In addition, a net result of changes in the $q, n$, and $T$ profile shapes is that the highest values of $f_{\mathrm{BS}}$ are at the mid-range value of $q_{\text {core }}$. Because of the changes in the density and temperature gradients with $q_{\text {core }}$ and $\beta_{\mathrm{N}}$, and the scaling of $J_{\mathrm{BS}}$ with the local value of $q$, equation (1) is not a good predictor of $f_{\mathrm{BS}}$ over the range of parameters of the discharges in this experiment. A scaling function of the form in equation (4) provides a much more complete description. 
The relatively uniform $J_{\mathrm{BS}}$ profiles in the region inside the H-mode pedestal in the highest $\beta_{\mathrm{N}}$ discharges are not a good match to the peaked profile of $J$ in the weak shear discharges produced for this experiment. In addition, $J_{\mathrm{BS}}$ is only a small fraction of $J$ in the innermost portion of the discharge (e.g. $\hat{\rho}<0.5)$. Operation at reduced $\beta_{\mathrm{N}}$ is not preferable, even though the $J_{\mathrm{BS}}$ profiles would be more peaked, because $f_{\mathrm{BS}}$ and fusion gain increase with $\beta_{\mathrm{N}}$. Therefore, externally driven noninductive current must be provided with a peaked profile so that $J_{\mathrm{NI}}$ is sufficiently peaked. In steady-state, if there is insufficient $J_{\mathrm{NI}}$ near the axis, the result would be a $q$ profile with negative central shear that could result in an ITB with a large local pressure gradient that would result in a low $\beta_{\mathrm{N}}$ stability limit.

Of the discharges produced for this experiment, the three with $q_{95}=6.8$ are reasonable candidates for reproducible $f_{\mathrm{NI}}=1$ operation in DIII-D with the present, or soon to be available, external current drive capability. Two of the three discharges had $f_{\mathrm{BS}} \approx 0.5$ and all three had $f_{\mathrm{NI}} \approx 0.8$. In the two discharges with $q_{\text {core }} \geq 1.43$, in order to increase $f_{\mathrm{NI}}$ to 1 , additional external current drive of $\approx 20 \mathrm{~A} \mathrm{~cm}^{-2}$ is required in the region $0.2<\hat{\rho}<0.7$. This additional current drive could be provided by the DIII-D ECCD system or by the planned off-axis neutral beam injection [37], preferably along with a reduction in $n_{\mathrm{e}}$ (figure 17) to increase their effectiveness. In the discharge with $q_{\text {core }} \approx 1.1$, the total additional externally driven current that is needed is the same as in the two higher $q_{\text {core }}$ discharges, but the required current density is 2 to 3 times higher and it must be located near the axis. As ECCD is more efficient on axis than off axis, this required noninductive current could also be provided by the DIII-D ECCD system [38].

The small value of $f_{\mathrm{BS}} \approx 0.4$ observed in this experiment at $q_{95} \approx 5$ is not sufficient for practical steady-state operation. However, for application to the steady-state scenario of ITER or 
to a reactor, operation at $q_{95} \approx 5$ is thought $[3,13,39,40]$ to be necessary in order to satisfy the requirement on the figure of merit $\beta_{\mathrm{N}} H_{98} / q_{95}^{2}$. At the lower values of $q_{95}$ the additional noninductively-driven current required for $f_{\mathrm{NI}}=1$ must be located off-axis (figure 16). Therefore it is useful to examine what the experimental results discussed here indicate about ways to increase $J_{\mathrm{BS}}$ off-axis and $f_{\mathrm{BS}}$ in order to make reproducible $f_{\mathrm{NI}}=1$ operation possible in DIII-D and future tokamaks at $q_{95} \approx 5$.

The primary paths to higher $f_{\mathrm{BS}}$ at fixed $q_{95}$ are increased $\beta_{\mathrm{N}}$, increased $q_{\text {core }}$, or increased gradients [equation (4)]. There are disadvantages that accompany each of these options and it is not obvious what path toward higher $f_{\mathrm{BS}}$ at $q_{95} \approx 5$ is the optimum. Pressure driven instabilities will set a maximum possible value of $\beta_{\mathrm{N}}$. The maximum $\beta_{\mathrm{N}}$ in the discharges discussed here was only $5 \%-25 \%$ below the calculated ideal-wall $n=1$ stability limit (figure 2), so only a small increase in $f_{\mathrm{BS}}$ would be anticipated by increasing $\beta_{\mathrm{N}}$ to the calculated limit. The projected $f_{\mathrm{BS}}$ is 0.55 in the best case. The $\beta_{\mathrm{N}}$ stability limit could be increased through a reduction [6] in the average peaking factor for the total pressure, which was approximately 3.3 in these discharges. The natural broadening of the thermal pressure profile as $\beta_{\mathrm{N}}$ is increased should help maintain stability, so it is possible that the calculated values in figure 2 are not the actual limit that would be encountered as $\beta_{\mathrm{N}}$ is increased beyond what was achieved in this experiment. In addition, there would be significant broadening of the total pressure profile if the fast ion pressure distribution could be broadened, through off-axis injection for instance, to match the profile of the thermal pressure for which the peaking factor is only 2.6 (figure 8 ). Broadening of the pressure profile should also have the effect of moving the peak density and temperature 
gradients to larger $\hat{\rho}$ thus increasing $J_{\mathrm{BS}}$ off-axis, the region where increased noninductive current is required for operation at reduced $q_{95}$.

At high $\beta_{\mathrm{N}}, J_{\mathrm{BS}}<J$ in the region near the axis (e.g. $\hat{\rho}<0.3$ ), so the value of $q_{\text {core }}$ will be controllable with the external current drive sources. A relatively large target value of $q_{\text {core }}$ would be preferable because that would reduce the demand for external current drive as well as increasing $J_{\mathrm{BS}}$. Reduced need for $J_{\mathrm{NBCD}}$ in the core region is compatible with off-axis beam injection to broaden the fast ion pressure profile. An increase in $q_{\min }$, though, tends to lead to a reduction in the maximum stable $\beta_{\mathrm{N}}[6]$, which would need to be offset with broader pressure profiles. To control $q_{\text {core }}$ when the external current drive is primarily $J_{\mathrm{NBCD}}$, it is necessary to have self-consistent values of the target value of $q_{\text {core }}, B_{\mathrm{T}}$ (which determines $J$ near the axis for a given $q_{\text {core }}$ ), neutral beam injected power and location (which determines the magnitude of $J_{\mathrm{NBCD}}$ near the axis), and target $\beta_{\mathrm{N}}$ (which determines the required neutral beam power which will vary with $B_{\mathrm{T}}$ ). Access to high $\beta_{\mathrm{N}}$ using primarily neutral beam heating may require offaxis deposition in order to avoid too much $J_{\mathrm{NBCD}}$ near the axis.

An increase in the density or temperature gradient will result in increased local $J_{\mathrm{BS}}$, but will also increase the pressure gradient and reduce the $\beta_{\mathrm{N}}$ stability limit. In addition, the experiment showed that the temperature and density gradients actually decrease as $\beta_{\mathrm{N}}$ is increased. So, paths to higher $f_{\mathrm{BS}}$ by increasing the gradients may be limited. A focus on reducing $n_{\mathrm{e}}$ and increasing $T_{\mathrm{e}}$ could be preferable. DIII-D discharges discussed previously [14,40,41] with $f_{\mathrm{NI}} \approx 1$ achieved $f_{\mathrm{BS}}$ higher than in this experiment because $n_{\mathrm{e}}$ was lower with comparable or larger $\left|\partial n_{\mathrm{e}} / \partial \rho\right|$ and $T_{\mathrm{e}}$ was higher with a broader profile. This resulted in more bootstrap current from the density gradient terms in equation (2). 
In conclusion, because of the broadening of the pressure profile as $\beta_{\mathrm{N}}$ is increased, operation in weak-shear-type steady-state discharges should optimize at relatively high $q_{\text {core }}$. High $q_{\text {core }}$ minimizes the external current drive requirement near the axis by reducing $J$ and increasing $J_{\mathrm{BS}}$ in that region. Broadening of the pressure profiles facilitates stable operation at the substantially increased $\beta_{\mathrm{N}}$ that is necessary to increase $f_{\mathrm{BS}}$ at the lower $q_{95}$ values. In addition, the density and temperature gradients, and thus $J_{\mathrm{BS}}$, will move further off axis where the noninductive current needs to be located for $q_{95} \approx 5$ discharges. Additional experiments are required to determine if a stable equilibrium can actually be produced at sufficiently high $\beta_{\mathrm{N}}$ with the required $J_{\mathrm{BS}}$ profile. The anticipated heating and current drive requirements are consistent with the plan to increase the off-axis neutral beam and ECCD powers available for DIII-D experiments. In neutral beam heated discharges, off-axis injection can aid in avoidance of excess $J_{\mathrm{NBCD}}$ near the axis and in broadening of the fast ion pressure profile while driving current offaxis where it is needed. ECCD drives current off-axis and provides electron heating that should increase the $T_{\mathrm{e}} \partial n_{\mathrm{e}} / \partial \rho$ term of $J_{\mathrm{BS}}$ that was relatively small in this experiment. 


\section{Appendix}

The idea that, in a scaling relation, $f_{\mathrm{BS}}$ should be treated as the sum of two terms, one proportional to $q_{\text {core }}$ and one proportional to $q_{95}$ is suggested by the data in figure 10(a). In that figure, $J_{\mathrm{BS}}$ is shown to be proportional to $q_{\text {core }}$ in the innermost portion of the discharge and $q_{95}$ in the outermost portion of the discharge when there are no changes in the density and temperature profiles. So, it should be possible to write $J_{\mathrm{BS}}$ as the sum of two functions, one which is proportional to $q_{\text {core }}$ which is large in the innermost portion of the discharge and small in the outermost portion of the discharge, and one which is proportional to $q_{95}$ which is small in the innermost portion of the discharge and large in the outermost portion. The total bootstrap current, $I_{\mathrm{BS}}$, calculated from the integral over the discharge cross-section of $J_{\mathrm{BS}}$, will then be the sum of a term proportional to $q_{\text {core }}$ and a term proportional to $q_{95}$, as will $f_{\mathrm{BS}}$.

By employing some approximations, each of the four terms in equation (2) can be rewritten as the sum of terms proportional to $q_{\text {core }}$ and $q_{95}$. Term $j(1 \leq j \leq 4)$ is written in the form

$$
\left\langle\left\langle J_{\mathrm{BSj}} B\right\rangle\right\rangle \approx \frac{-R_{0}}{\rho} \frac{\langle x y\rangle g(\rho)}{l} \mathcal{L}(\rho) q(\rho),
$$

where $x(\rho)$ and $y(\rho)$ are the appropriate temperature or density, $l$ is a constant radial scale length for $y(\rho), g(\rho)$ is a normalized function representing the pressure profile shape, and $B_{\mathrm{T} 0} R_{0} / F=1$ has been used. The $q$ profile is parameterized as $q(\rho)=q_{\text {core }} f_{\text {core }}(\rho)+q_{95} f_{95}(\rho)$. For instance, good fits to the $q$ profiles in this experiment can be made using $q_{\text {min }}$ instead of $q_{\text {core }}$ and $f_{\text {core }}=\left(1-\rho^{\alpha_{1}}\right)^{\alpha_{2}}$ and $f_{95}=\rho^{2.8}$. Similarly, the assumption is made that the pressure profile shape can be parameterized as 
$g(\rho) / l=f_{\mathrm{p}}^{\alpha_{\text {core }}} g_{\text {core }}(\rho)+f_{\mathrm{p}}^{\alpha_{95}} g_{95}(\rho)$. The values of $q_{\text {core }}, q_{95}$, and $f_{\mathrm{p}}$ are specific to a given discharge. The assumption is made that these values adequately characterize the discharges so that $\mathcal{L}(\rho), f_{\text {core }}(\rho), f_{95}(\rho), g_{\text {core }}(\rho)$, and $g_{95}(\rho)$ can be treated as the same for all discharges. Products of the functions representing the inner and outer portions of the discharge are ignored as small.

The area integral of term $j$ is

$$
\begin{aligned}
1_{\mathrm{BSj}} / I_{\mathrm{p}} & =\frac{1}{I_{\mathrm{p}}} \int 2 \pi \rho d \rho B_{\mathrm{T} 0} \frac{\left.\left\langle J_{\mathrm{BSj}} B\right\rangle\right\rangle}{\left\langle\left\langle B^{2}\right\rangle\right\rangle} \\
& \propto-R_{0} \beta_{\mathrm{Nth}}^{\mathrm{i}, \mathrm{e}}\left(f_{\mathrm{p}}^{\alpha_{\text {core }}} q_{\text {core }} \int_{0}^{1} \mathrm{~d} \hat{\rho} f_{\text {core }} g_{\text {core }} \mathcal{L}+f_{\mathrm{p}}^{\alpha_{95}} q_{95} \int_{0}^{1} d \hat{\rho} f_{95} g_{95} \mathcal{L}\right),
\end{aligned}
$$

where $B_{\mathrm{T} 0} /\left\langle\left\langle B^{2}\right\rangle\right\rangle \approx 1 / B_{\mathrm{T} 0}$ and $\rho_{\mathrm{b}} / a=$ constant have been used and $\beta_{\mathrm{N}}^{\mathrm{i}, \mathrm{e}}$ is $\beta_{\mathrm{N}}$ with the volume average plasma pressure replaced by the volume average ion or electron thermal pressure. Thus, the bootstrap current fraction resulting from each of the terms in equation (2) has been written in the form of equation (4). The assumption is made that a fitting function of the same form can describe the total $f_{\mathrm{BS}}$.

\section{Acknowledgment}

This work was supported in part by the US Department of Energy under DE-FC0204ER54698, DE-AC52-07NA27344, DE-AC05-06OR23100, and DE-FG02-08ER54984. 


\section{References}

[1] Bickerton R.J. et al 1971 Nature Physical Science 229110

[2] Luce T.C. 2010 "Realizing steady-state tokamak operation for fusion energy," submitted to Phys. Plasmas

[3] Gormezano C. et al 2007 Nucl. Fusion 47 S285

[4] Waltz R.E. et al 1995 Phys. Plasmas 22408

[5] Petty C.C. et al 1998 Phys. Plasmas 51695

[6] Ferron J.R. et al 2005 Phys. Plasmas 12056126

[7] Hender T.C. et al 2007 Nucl. Fusion 47 S128

[8] Cordey J.G. et al 1988 Plasma Phys. Control. Fusion 301625

[9] Wilson H.R 1992 Nucl. Fusion 32257

[10] Andrade M.C.R and Ludwig G.O. 2008 Plasma Phys. Control. Fusion 50065001

[11] Garofalo A.M. et al 2006 Phys. Plasmas 13056110

[12] Coda S. et al 2005 Phys. Plasmas 12056124

[13] Murakami M. et al 2006 Phys. Plasmas 13056106

[14] Holcomb C.T. et al 2009 Phys. Plasmas 16056116

[15] Suzuki T. et al 2009 Nucl. Fusion 49085003

[16] Sakamoto Y. et al 2009 Nucl. Fusion 49095017

[17] Oyama N. and the JT-60 Team 2009 Nucl. Fusion 49104007

[18] Litaudon X. et al 2002 Plasma Phys. Control. Fusion 441057

[19] Voitsekhovitch I. et al 2009 Nucl. Fusion 49055026

[20] Wade M.R. et al 2003 Nucl. Fusion 43634

[21] Murakami M. et al 2005 Nucl. Fusion 451419 
[22] Ferron J.R. et al 2006 Nucl. Fusion 46 L13

[23] ITER Physics Expert Group on Confinement et al 1999 Nucl. Fusion 392175

[24] Lodestro L.L. and Pearlstein L.D. 1994 Phys. Plasmas 190

[25] Glasser A.H. and Chance M.S. 1997 Bull. Am. Phys. Soc. 421848

[26] Lao L.L. et al 1990 Nucl Fusion 301035

[27] St John H.E. et al 1995 Proc. $15^{\text {th }}$ Int. Conf. on Plasma Phys. and Control. Nucl. Research, Vol. 3, Seville, 1994 (IAEA, Vienna) p. 603

[28] Pankin A. et al 2004 Comput. Phys. Commun. 159157

[29] Rice B.W. et al 1997 Nucl. Fusion 37517

[30] Zarnstorff M.C. et al 1997 Phys. Plasmas 41097

[31] Sauter O. et al 1999 Phys. Plasmas 62834

[32] Sauter O. et al 2002 Phys. Plasmas 95140

[33] Lin Liu Y.R. et al 2003 Phys. Plasmas 104064

[34] Forest C.B. et al 1994 Phys. Rev. Lett. 732444

[35] Groebner R.J. et al 2009 Nucl. Fusion 49085037

[36] Bevington P.R. 1969 "Data Reduction and Error Analysis for the Physical Sciences," McGraw-Hill, p. 200

[37] Murakami M. et al 2009 Nucl. Fusion 49065031

[38] Petty C.C. et al 2009 Bull. Am. Phys. Soc. 54166

[39] Murakami M. et al 2010 Proc. of $23^{\text {rd }}$ Fusion Energy Conf., Daejeon, Republic of Korea (IAEA) ITR/P1-35. 
[40] Park J.M. et al 2010 Proc. of $23^{\text {rd }}$ Fusion Energy Conf., Daejeon, Republic of Korea (IAEA) EXC/P2-05

[41] Doyle E.J. et al 2010 Nucl. Fusion 50075005 


\section{Figure Captions}

FIG. 1. Profiles of the safety factor $(q)$ in the set of discharges with $\beta_{\mathrm{N}} \approx 2.8$, averaged over the high $\beta_{\mathrm{N}}$ phase of the discharge. The color of the curves indicates the value of $q_{95}: 4.5$ (red), 5.6 (blue), 6.8 (green). The line type indicates the nominal value of $q_{\min }: 1.0$ (solid), 1.5 (dashed), 2.0 (dot-dash).

FIG. 2. The $\beta_{\mathrm{N}}$ value in discharges with the neutral beam power at $13.5 \mathrm{MW}$, the maximum available (solid symbols). The open symbols are the ideal $n=1$ stability limit calculated by scaling the pressure profile from the reconstructed equilibria for the experimental discharges using the TEQ [24] and DCON [25] codes. The lower set of open symbols is the stability limit calculated without a conducting wall and the upper set of symbols was calculated with an ideal wall with the shape of the DIII-D limiter. The lines connect points with the same value of $q_{95}$ : 4.5 (red triangles), 5.6 (blue squares), 6.8 (green circles).

FIG. 3. For the series of discharges at $\beta_{\mathrm{N}}=2.8$, (a) electron temperature and (b) electron temperature gradient as a function of the normalized radius. In (a), the arrows highlight the increase in temperature at mid-radius that indicates the broadening of the profiles with increases in $q_{\mathrm{min}}$. The error bars indicate the standard deviation of the multiple profile measurements during the $\beta_{\mathrm{N}} \approx 2.8$ phase of the discharge that were averaged to produce the curves shown. The color of the curves indicates the value of $q_{95}: 4.5$ (red), 5.6 (blue), 6.8 (green). The line type indicates the approximate value of $q_{\min }: 1.0$ (solid), 1.5 (dashed), 2.0 (dot-dash).

FIG. 4. For the series of discharges at $\beta_{\mathrm{N}}=2.8$, (a) ion temperature and (b) ion temperature gradient as a function of the normalized radius. The line types and colors are as described for figure 3 . 
FIG. 5. For the series of discharges with the maximum available neutral beam power, (a) electron temperature and (b) ion temperature as a function of the normalized radius. The line types and colors are as described for figure 3 .

FIG. 6. For the series of discharges at $\beta_{\mathrm{N}}=2.8$, (a) electron density and (b) electron density gradient as a function of the normalized radius. The line types and colors are as described for figure 3 .

FIG. 7. For the series of discharges with the maximum available neutral beam power, (a) electron density and (b) electron density gradient as a function of the normalized radius. The line types and colors are as described for figure 3 .

FIG. 8. The peaking factor for the thermal pressure, as defined in equation (3), for all of the $\beta_{\mathrm{N}} \approx 2.8$ (open symbols) and maximum neutral beam power (closed symbols) discharges. The dashed lines highlight the trends. The symbol types and colors indicate the value of $q_{95}: 4.5$ (red triangles), 5.6 (blue squares), 6.8 (green circles).

FIG. 9. Profiles of the bootstrap current density computed from equation (2), the measured density and temperature profiles and the reconstructed equilibria for (a) the discharges with $\beta_{\mathrm{N}} \approx 2.8$, (b) the discharges heated with the maximum available neutral beam power. The line types and colors are as described for figure 3. 
FIG. 10. Profiles of (a) bootstrap and (b) neutral beam driven current density computed from models using the measured $q$ profiles from the discharges with $\beta_{\mathrm{N}} \approx 2.8$. In all cases, the density and temperature profiles were those measured for the $q_{\min } \approx 1.5, q_{95}=5.6, \beta_{\mathrm{N}} \approx 2.8$ discharge so that these results show only the effect of changing the $q$ profile. The line types and colors are as described for figure 3 .

FIG. 11. Calculated fraction of the total plasma current resulting from bootstrap current $\left(f_{\mathrm{BS}}\right)$ for discharges with $\beta_{\mathrm{N}}=2.8$ (open symbols) and discharges heated by the maximum available neutral beam power in order to maximize $\beta_{\mathrm{N}}$ (closed symbols). The lines connect points with the same value of $q_{95}: 4.5$ (red triangles), 5.6 (blue squares), 6.8 (green circles).

FIG. 12. Ratio of the bootstrap current fraction to the normalized beta computed from the thermal component of the pressure [equation (3)]. The colors and symbol shapes represent the nominal value of $q_{\text {core }}: 1$ (magenta triangles), 1.5 (green squares), $\approx 2$ (black circles). Open symbols are for discharges with $\beta_{\mathrm{N}} \approx 2.8$ and closed symbols are for discharges with the maximum neutral beam power. The dashed line is a fit to the data shown as open symbols which has an offset at $q_{95}=0$ equal to 0.095 . The dot-dashed line is a fit to the data shown as closed symbols with offset at $q_{95}=0$ equal to 0.049 .

FIG. 13. A fit of $f_{\mathrm{BS}} / \beta_{\mathrm{Nth}}$ to the function specified in equation (4). The values computed from the experimental data are plotted versus the values computed from the fitting function with $A=$ $0.0018 \pm 0.0015, B=0.052 \pm 0.045, \alpha_{\text {core }}=3.0 \pm 0.75$, and $\alpha_{95}=-0.94 \pm 0.94$. Open symbols are for discharges with $\beta_{\mathrm{N}} \approx 2.8$ and closed symbols are for discharges with the maximum neutral beam power. The colors indicate the value of $q_{95}: 4.5$ (red), 5.6 (blue), 6.8 (green). The symbol 
shapes represent the nominal value of $q_{\text {core }}: 1$ (triangles), 1.5 (squares), $\approx 2$ (circles).

FIG. 14. (a) The computed noninductive current fraction and (b) the fraction of current driven by the neutral beams. The open symbols are for the discharges with $\beta_{\mathrm{N}} \approx 2.8$ and the solid symbols are for the discharges heated by the maximum available neutral beam power. The lines connect points with the same value of $q_{95}: 4.5$ (red triangles), 5.6 (blue squares), 6.8 (green circles).

FIG. 15. Radial profiles of current density in discharges with maximum available neutral beam power, $q_{95}=6.8$ and different values of $q_{\text {core }}$ : (a) 1.1 , (b) 1.43 , (c) 1.77 . The total current density (dashed line) is taken from the equilibrium reconstruction, the bootstrap current density (solid line), neutral beam driven current density (long dashed line) and electron cyclotron driven current density (dotted line) are calculated from models. The total noninductive current density (dash-dot line) is $J_{\mathrm{NBCD}}+J_{\mathrm{ECCD}}+J_{\mathrm{BS}}$.

FIG. 16. Radial profiles of current density in the discharge with maximum available neutral beam power and $q_{95}=4.5$ and $q_{\text {core }} \approx 1.56$. The curve types correspond to the same values as described in the caption for figure 15.

FIG. 17. The computed current density profiles assuming the parameters of the maximum available neutral beam power discharge with $q_{95}=6.8$ and $q_{\text {core }} \approx 1.43$ but with a uniform $20 \%$ reduction in $n_{\mathrm{e}}$ and a corresponding increase in temperatures to keep $\beta_{\mathrm{N}}$ constant. The curve types correspond to the same values as described in the caption for figure 15. 


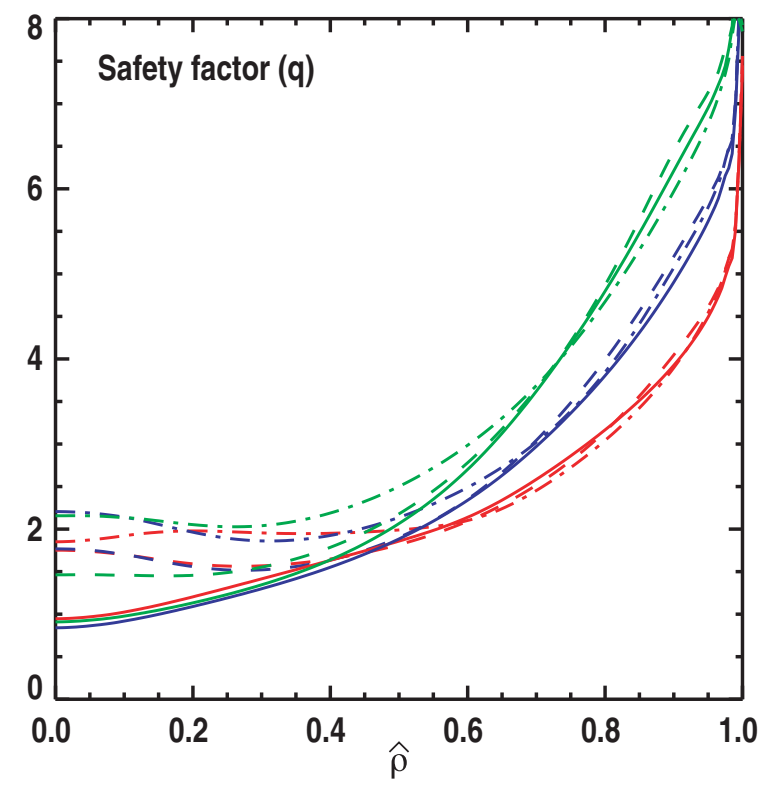

J.R. Ferron

Figure 1 


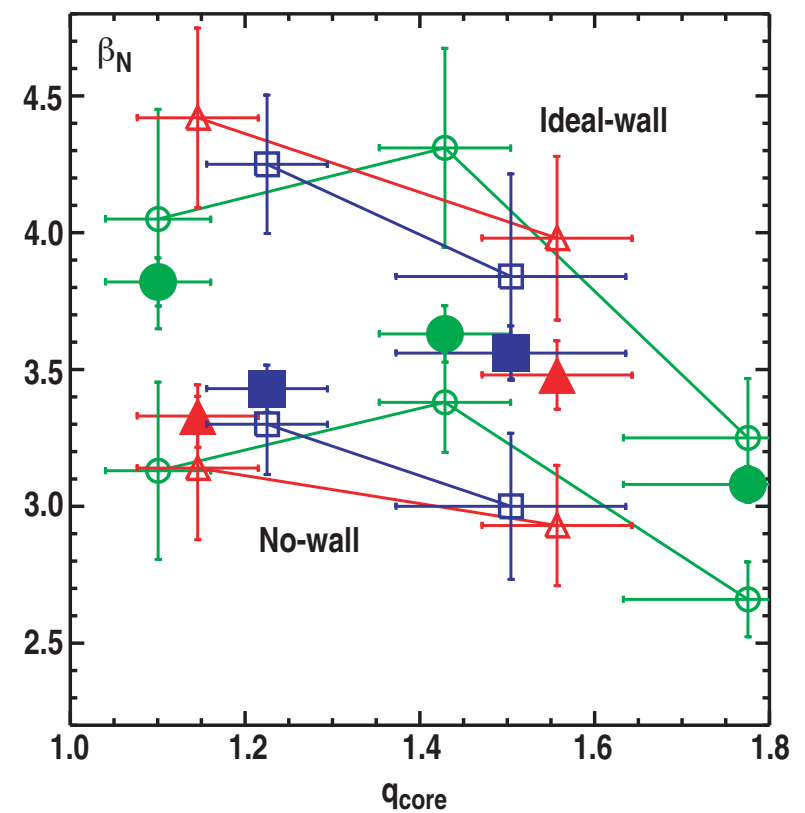

J.R. Ferron Figure 2 

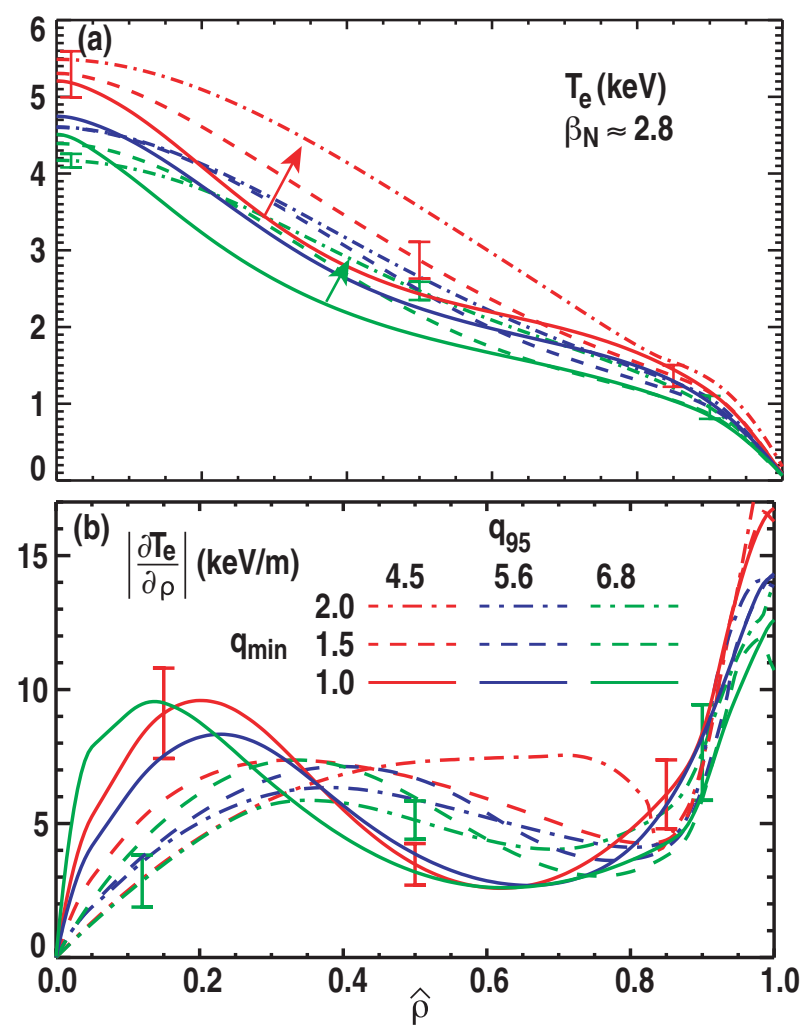

J.R. Ferron

Figure 3 

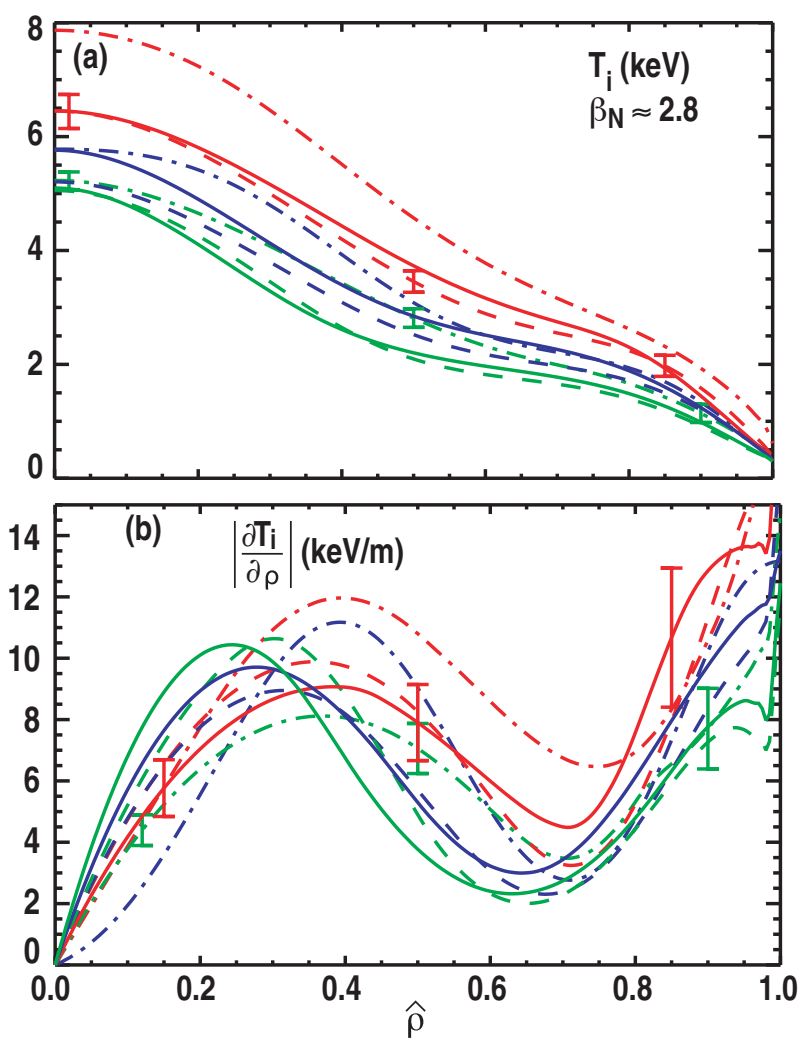

J.R. Ferron

Figure 4 


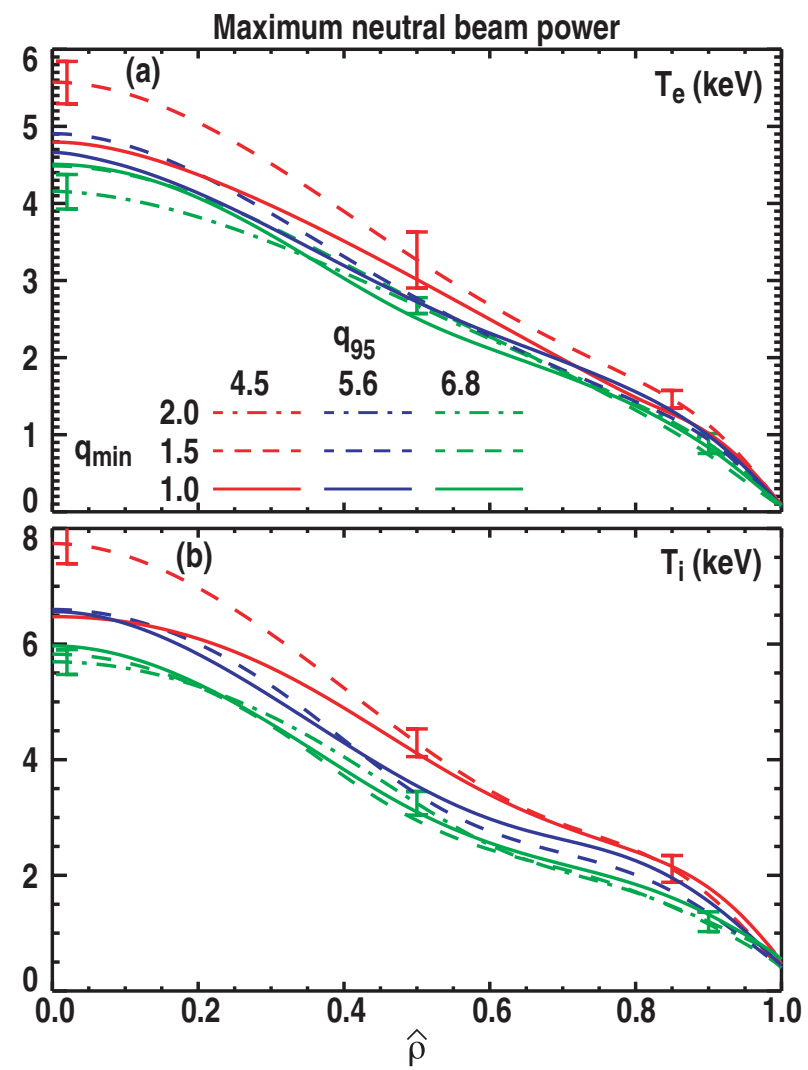

J.R. Ferron

Figure 5 

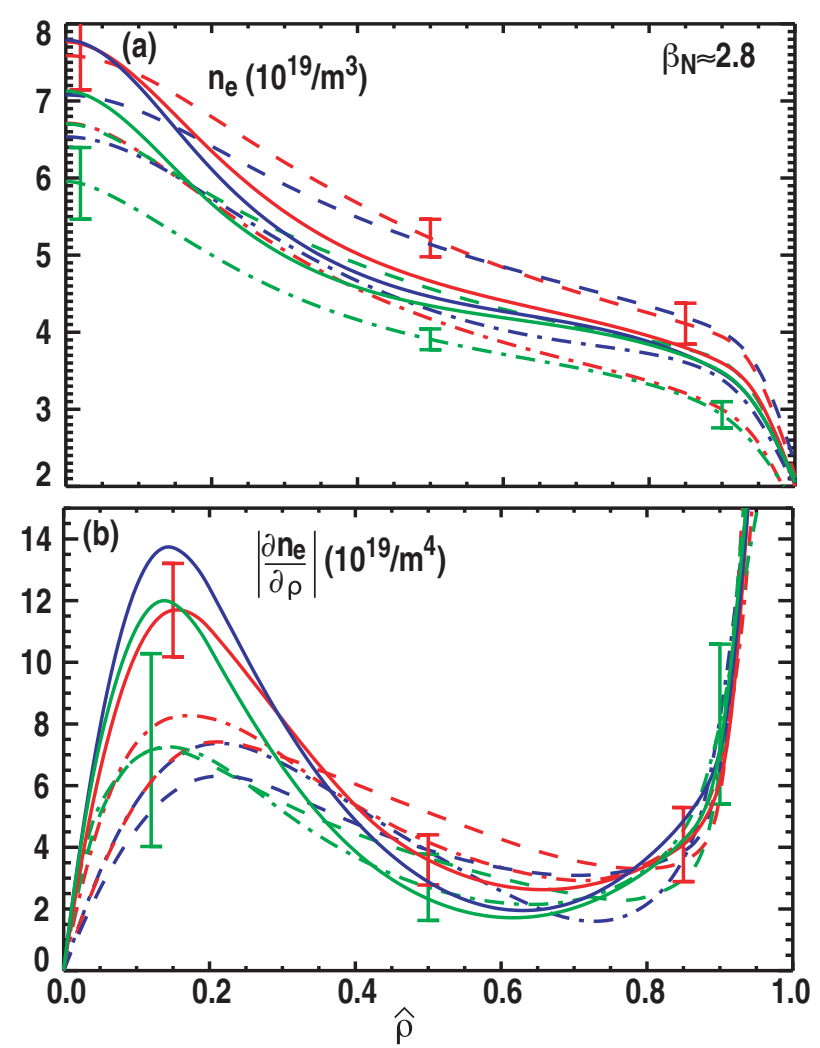

J.R. Ferron

Figure 6 

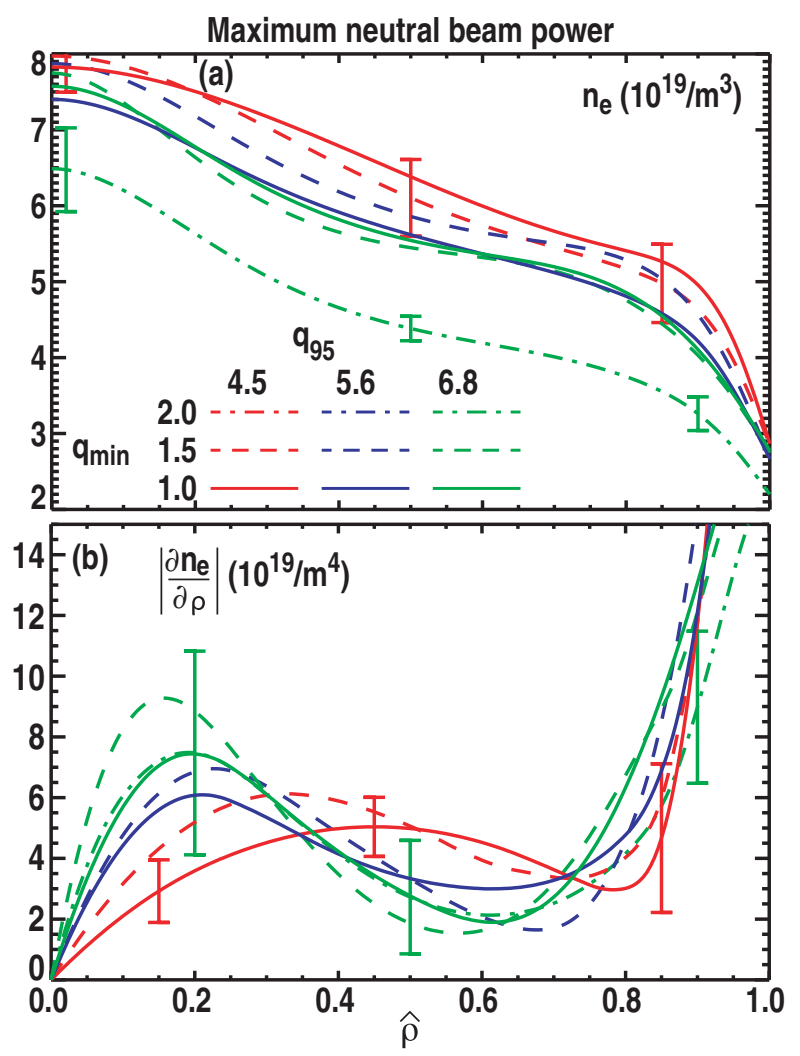

J.R. Ferron

Figure 7 


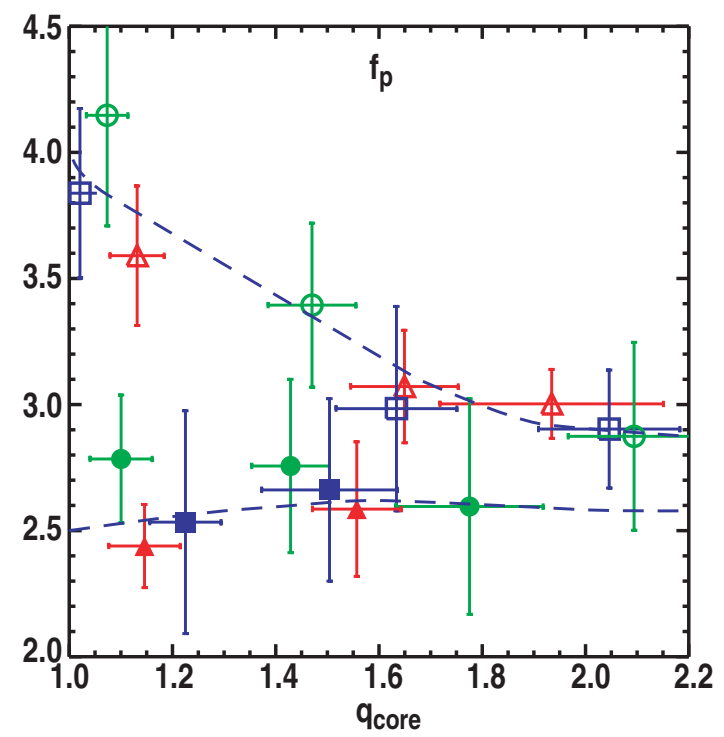

J.R. Ferron

Figure 8 

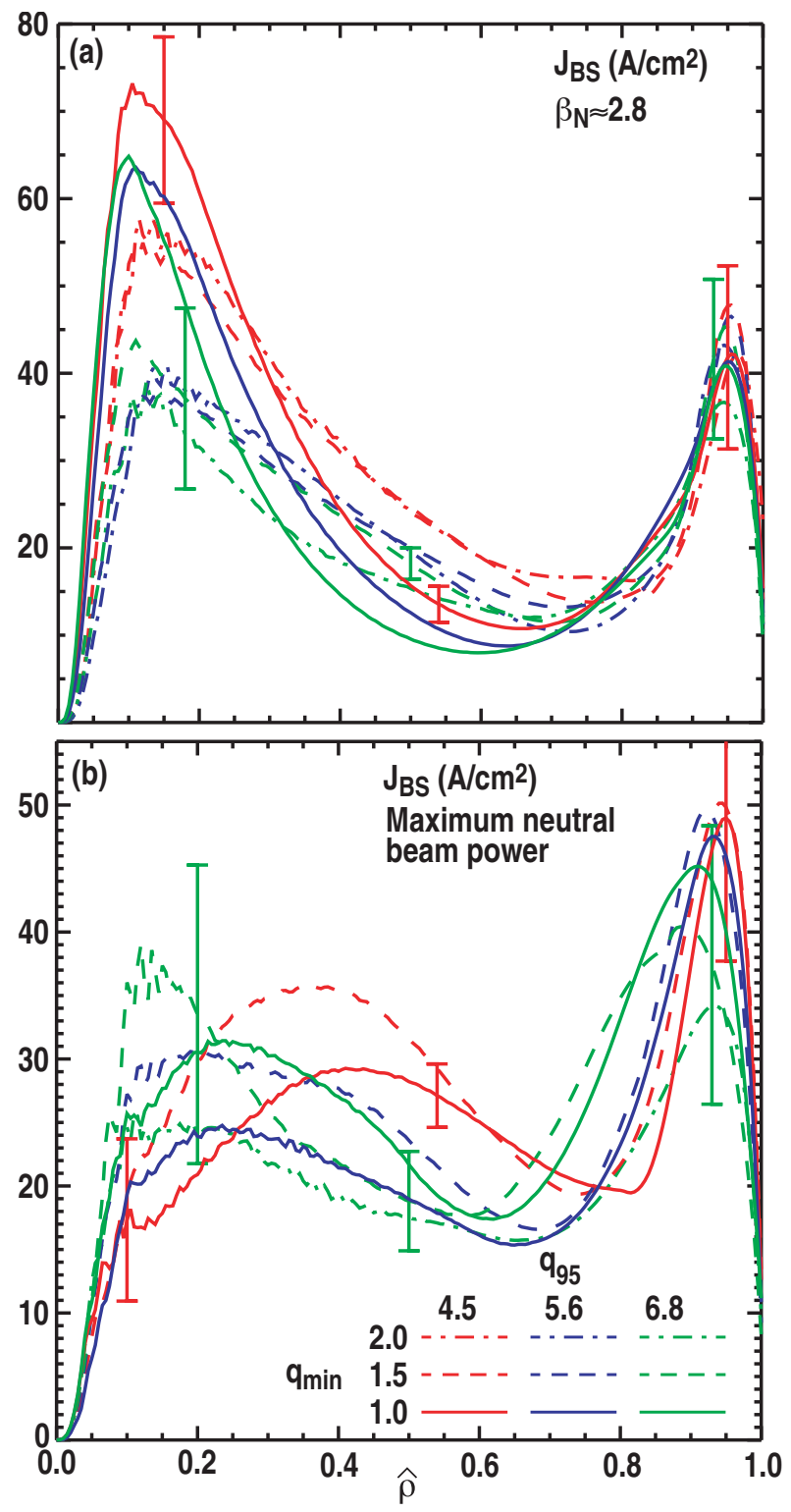

J.R. Ferron

Figure 9 

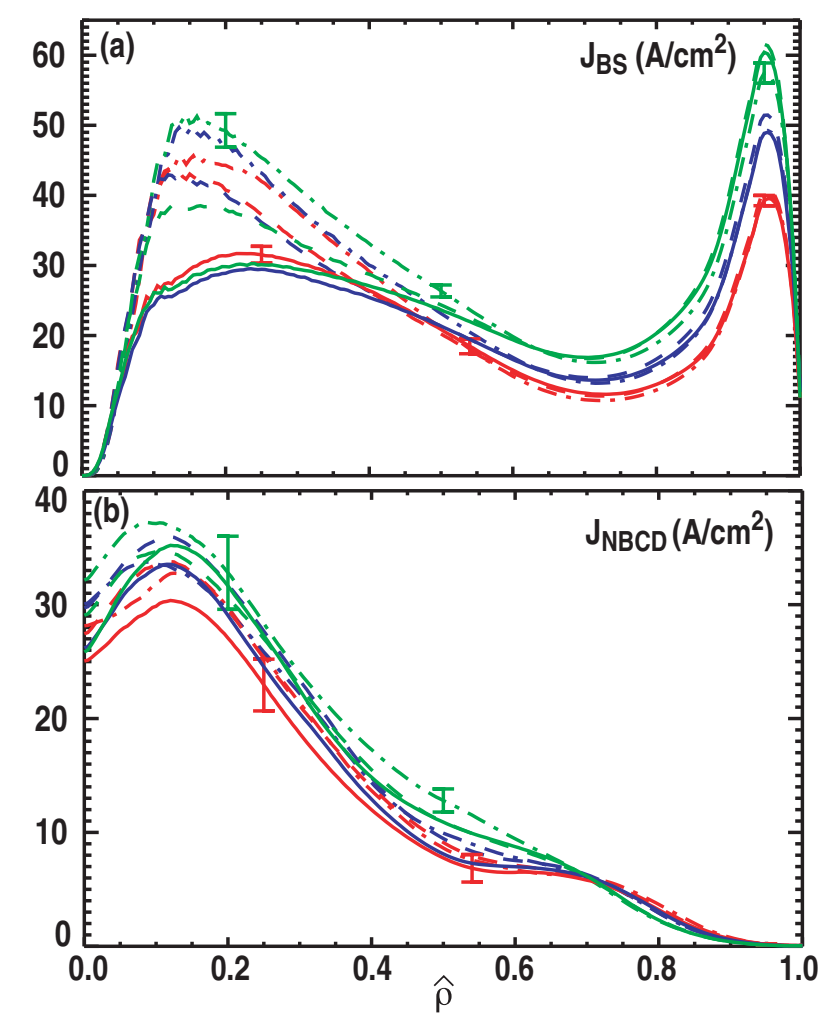

J.R. Ferron

Figure 10 


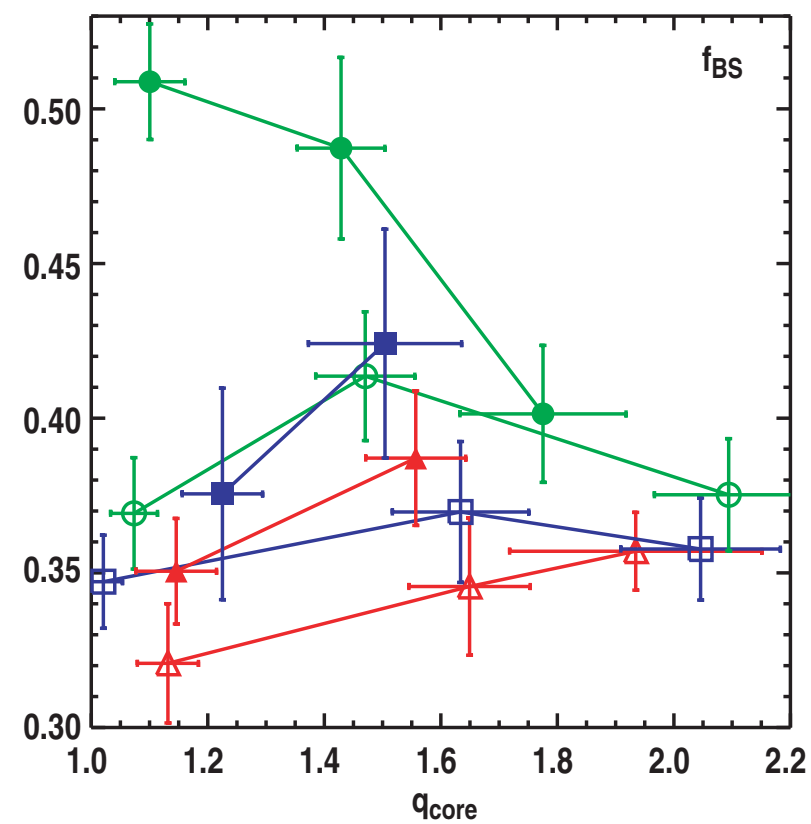

J.R. Ferron

Figure 11 


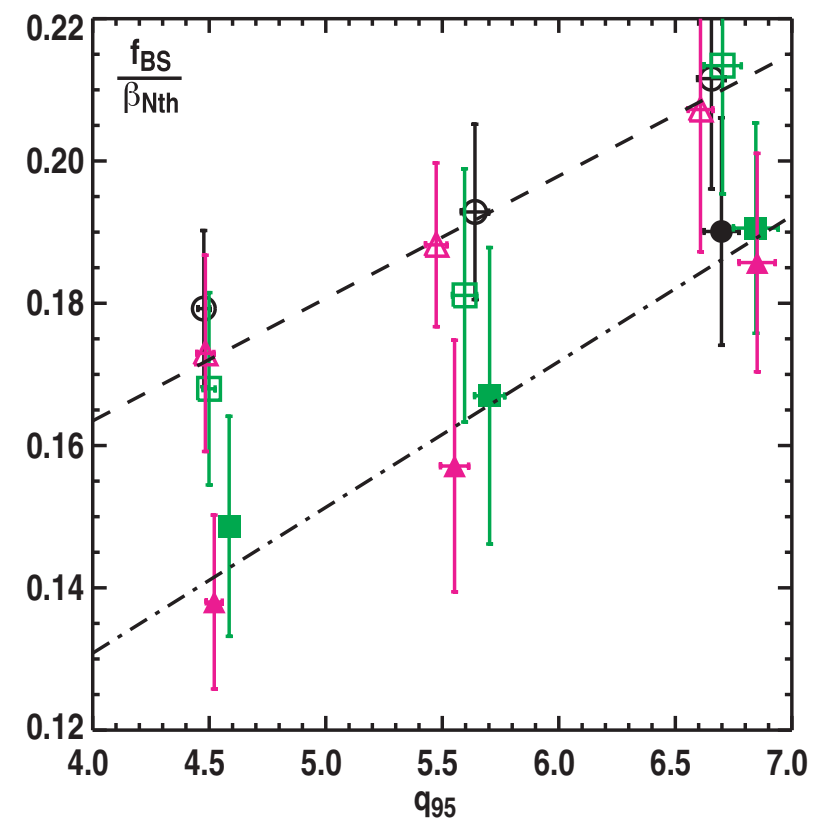

J.R. Ferron

Figure 12 


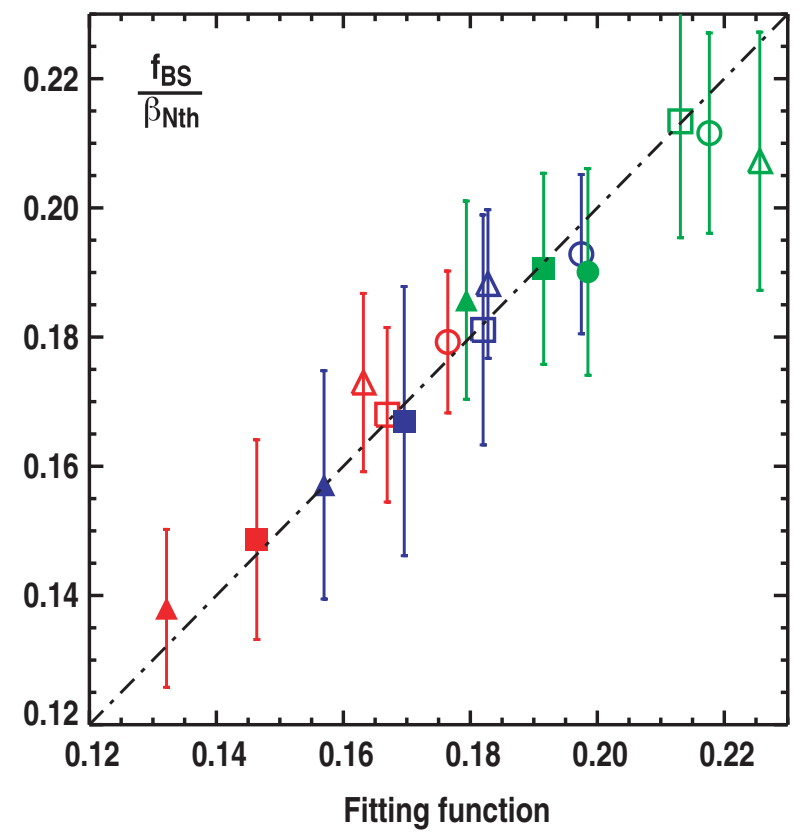

J.R. Ferron

Figure 13 

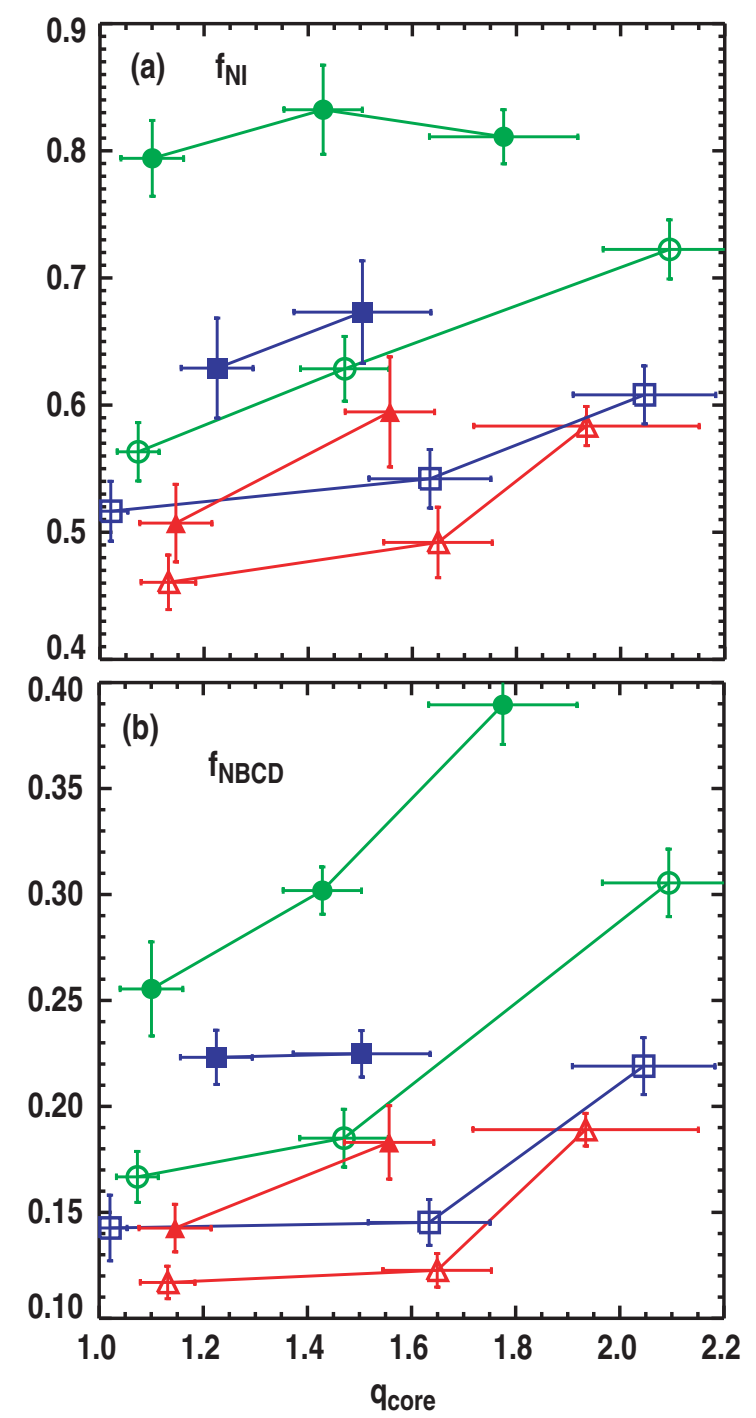

J.R. Ferron Figure 14 


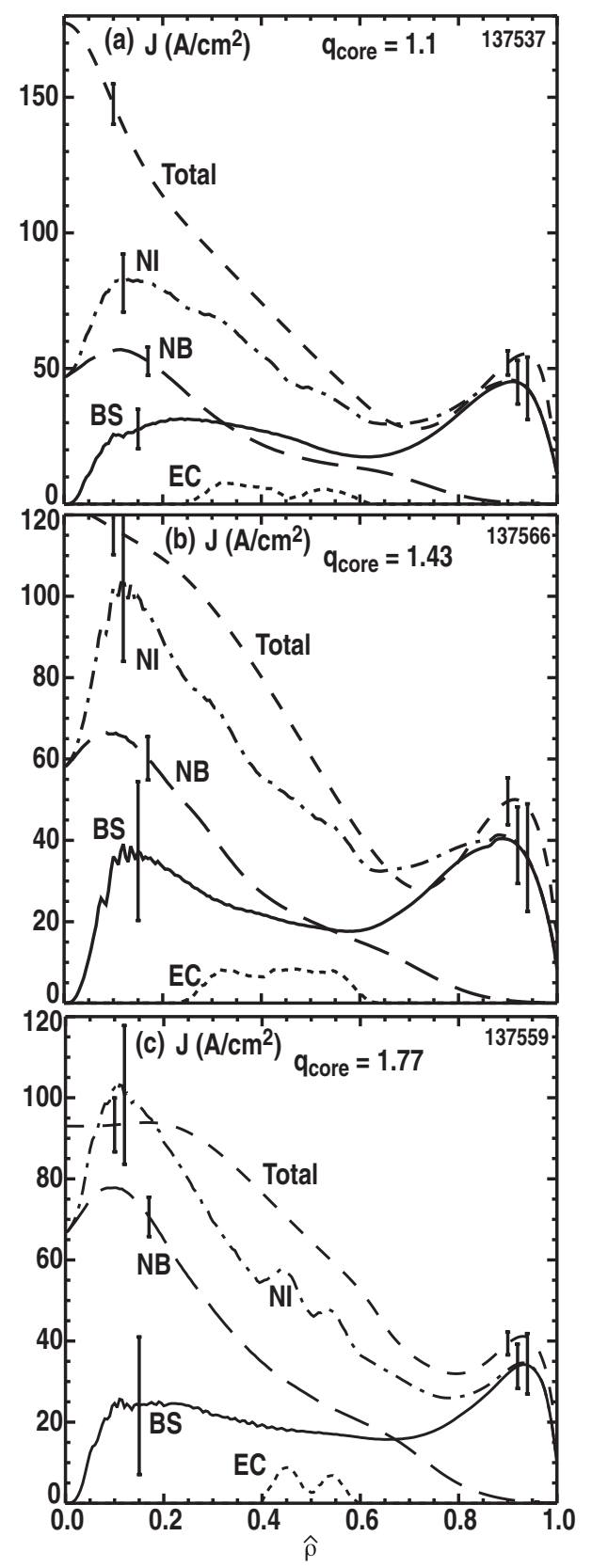

J.R. Ferron

Figure 15 


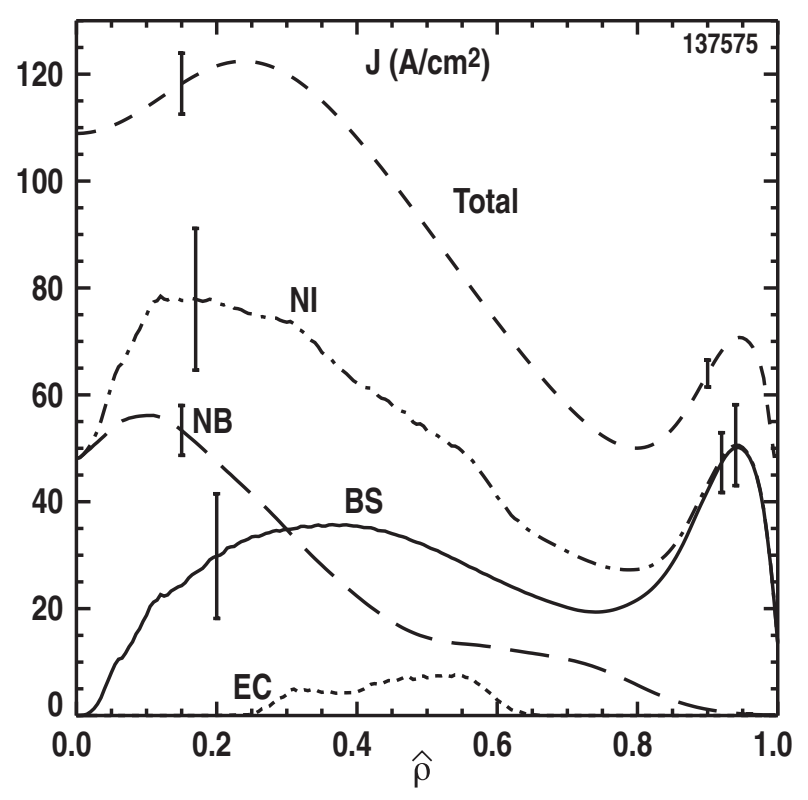

J.R. Ferron

Figure 16 


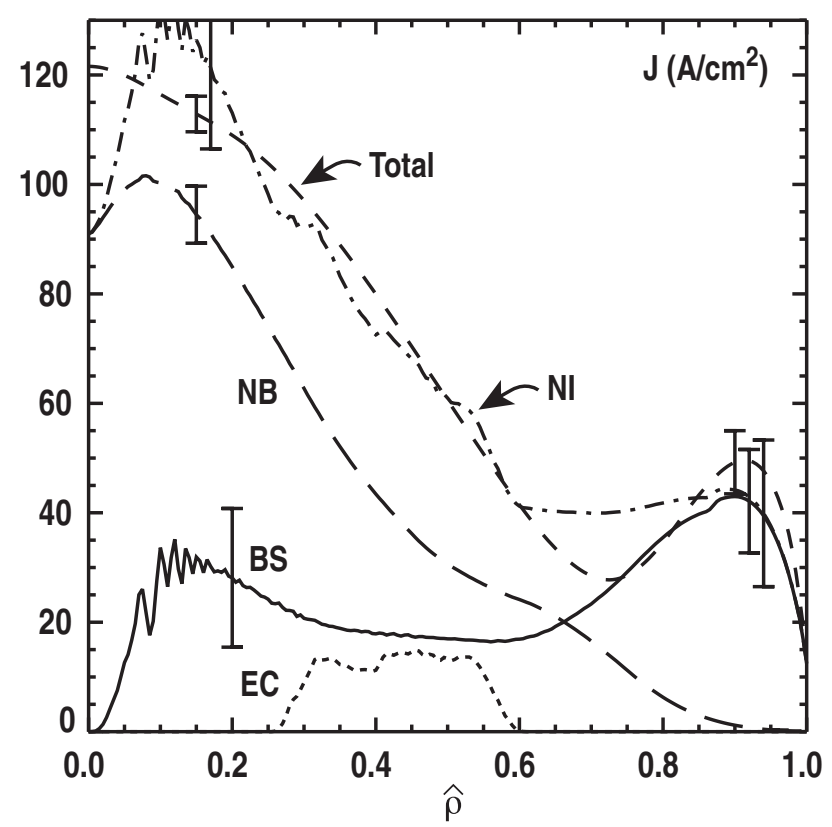

J.R. Ferron

Figure 17 